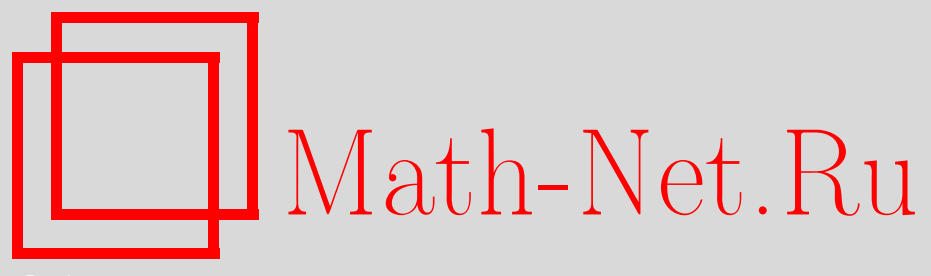

Д. А. Сапронов, А. Е. Шишков, Асимптотическое поведение носителей решений квазилинейных многомерных параболических уравнений типа нестационарной диффузии-конвекции, Матем. сб., 2006, том 197, номер 5, 125-160

DOI: https://doi.org/10.4213/sm1561

Использование Общероссийского математического портала Math-Net.Ru подразумевает, что вы прочитали и согласны с пользовательским соглашением http: //www . mathnet.ru/rus/agreement

Параметры загрузки:

IP : 3.80 .253 .173

26 апреля 2023 г., 10:46:03

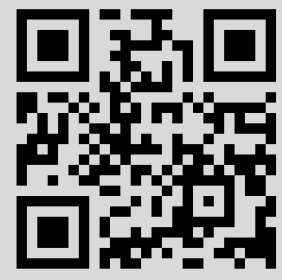




\author{
Д. А. Сапронов, А. Е. Шишков
}

\title{
Асимптотическое поведение носителей решений квазилинейных многомерных параболических уравнений типа нестационарной диффузии-конвекции
}

Изучается эффект конечности скорости распространения носителей обобщенных энергетических решений смешанных задач для широкого класса дважды вырождающихся параболических уравнений высокого порядка, модельным представителем которых является уравнение

$$
\left(|u|^{q-1} u\right)_{t}+(-1)^{m} \sum_{|\alpha|=m} D_{x}^{\alpha}\left(\left|D_{x}^{\alpha} u\right|^{p-1} D_{x}^{\alpha} u\right)+\left(|u|^{\lambda-1} u\right)_{x_{1}}=0,
$$

$m \geqslant 1, p>0, q>0, \lambda>0$.

Установлены в определенном смысле точные оценки начальной эволюции носителей решений (в частности, "правого" и “левого" фронтов носителей), зависящие от локальных свойств начальной функции и параметров уравнения. Исследовано также поведение носителей при больших значениях времени.

Библиография: 31 название.

\section{§ 1. Введение}

В области $G_{T}=(0, T) \times \Omega$, где $\Omega$ - произвольная (возможно, и неограниченная) область в $\mathbb{R}^{n}, n>1$, рассматривается задача Коши-Дирихле для следующего класса квазилинейных параболических уравнений порядка $2 m, m \geqslant 1$ :

$$
\begin{gathered}
P_{q}(u)+A_{p}^{(2 m)}(u)+B_{\lambda}(u) \\
\equiv\left(|u|^{q-1} u\right)_{t}+(-1)^{m} \sum_{|\alpha|=m} D_{x}^{\alpha} a_{\alpha}\left(t, x, u, D_{x} u, \ldots, D_{x}^{m} u\right) \\
+\chi \cdot \nabla b(t, u)=0, \quad q>0, \\
u(0, x)=u_{0}(x) \in L_{q+1}(\Omega), \\
\left.D_{x}^{\alpha} u\right|_{(0, T) \times \partial \Omega}=0, \quad|\alpha| \leqslant m-1 .
\end{gathered}
$$

Здесь $\chi=\left(\chi_{1}, \chi_{2}, \ldots, \chi_{n}\right)$ - произвольный вектор из $\mathbb{R}^{n}$, а непрерывные функции $a_{\alpha}(t, x, \xi)$ и $b(t, s)$ удовлетворяют условиям коэрцитивности и роста:

$$
\begin{gathered}
\sum_{|\alpha|=m} a_{\alpha}(t, x, \xi) \xi_{\alpha} \geqslant d_{0} \sum_{|\beta|=m}\left|\xi_{\beta}\right|^{p+1} \\
\forall(t, x, \xi) \in G \times \mathbb{R}^{N(m)}, \quad p>0, \quad d_{0}>0, \\
\left|a_{\alpha}(t, x, \xi)\right| \leqslant d_{1} \sum_{|\beta|=m}\left|\xi_{\beta}\right|^{p} \quad \forall(t, x, \xi) \in G \times \mathbb{R}^{N(m)}, \quad d_{1}<\infty,
\end{gathered}
$$




$$
\begin{array}{r}
|b(t, s)| \leqslant d_{2}|s|^{\lambda} \quad \forall(t, s) \in \mathbb{R}_{+}^{1} \times \mathbb{R}^{1}, \quad d_{2}<\infty, \quad \lambda>0, \\
b(t, s) s-\int_{0}^{s} b(t, \tau) d \tau \geqslant d_{3}|s|^{\lambda+1} \quad \forall(t, s) \in \mathbb{R}_{+}^{1} \times \mathbb{R}^{1}, \quad d_{3}>0
\end{array}
$$

где $N(m)$ - число различных $n$-мерных мультииндексов $\alpha=\left(\alpha_{1}, \alpha_{2}, \ldots, \alpha_{n}\right)$ длины $|\alpha|=\alpha_{1}+\alpha_{2}+\cdots+\alpha_{n} \leqslant m$.

Уравнения структуры (1.1) при $m=1$ моделируют нелинейные диффузноконвективные процессы. Модельный пример - одномерное уравнение ньютоновской фильтрации с конвективным переносом вдоль положительного направления оси $o x_{1}$ :

$$
\begin{gathered}
\left(|u|^{q-1} u\right)_{t}-u_{x x}+\chi\left(|u|^{\lambda-1} u\right)_{x}=0, \quad(t, x) \in(0, T) \times \mathbb{R}^{1}, \quad 0<\chi=\mathrm{const} \\
u(0, x)=u_{0}(x), \quad 0 \leqslant u_{0}(x) \in C^{0}\left(\mathbb{R}^{1}\right) .
\end{gathered}
$$

Уравнение (1.8) при $q<1$ (уравнение медленной диффузии) моделирует, к примеру, фильтрацию жидкости через однородную пористую среду, теплопроводность, теплоемкость и теплоперенос в которой зависят от температуры в каждой ее точке степенным образом, течение тонкой вязкой пленки по наклонной плоскости, диффузию газа, при $q>1$ (уравнение быстрой диффузии) - перенос термальной энергии в плазме и т.д. Ряд физических моделей (эволюция тонких пленок, бинарных расплавов) приводит к квазилинейным вырождающимся параболическим уравнениям высокого (в частности, четвертого) порядка, близким к (1.1).

Уравнения структуры (1.1) являются вырождающимися (“дважды" вырождающимися при $q \neq 1, p \neq 1)$ квазилинейными параболическими уравнениями. Изучение разрешимости граничных задач для этих уравнений было начато в известной статье О.А. Олейник, А. С. Калашникова, Чжоу Юй-линь [1], в которой введено понятие непрерывного обобщенного решения задачи Коши для уравнения структуры (1.8) с $\chi=0$ и установлены его существование и единственность. В дальнейшем построению теории вырождающихся параболических уравнений было посвящено огромное количество работ (см. обзор [2], книгу [3] и библиографию к ним). Так, разрешимость смешанных задач для одномерных уравнений ньютоновской фильтрации с нелинейной конвекцией изучалась в [4]-[7].

Хорошо известна основанная на методе монотонности теория разрешимости граничных задач для общих уравнений (1.1) с $q=1, B_{\lambda}(u)=0$ (см. книги [8], [9]). Некоторые результаты о разрешимости "дважды" вырождающихся уравнений структуры (1.1) с $q \neq 1, p \neq 1$ при наличии младших членов типа нелинейной абсорбции установлены в [10], [11]. Существование и единственность слабого решения задачи Коши-Дирихле в ограниченной области с нулевым граничным условием для уравнения многомерной фильтрации с конвекцией при некоторых дополнительных ограничениях на показатели нелинейности были установлены в [12], а в [13] - однозначная разрешимость этой задачи для более широкого класса параболических уравнений второго порядка, в который входит и уравнение (1.1) при $m=1$. Кроме того, в работе [13] установлены также близкие к (1.4)-(1.7) естественные ограничения на нелинейности уравнения, при которых слабое решение является энергетическим.

Одним из характерных свойств решений вырождающихся параболических уравнений (уравнение (1.8) вырождается при $u=0$ ) является свойство конечности скорости распространения носителей их решений. Так, при

$$
\operatorname{supp} u_{0}(x)=[-a, a], \quad 0<a<\infty, \quad q<1, \quad \lambda>q
$$


носитель произвольного решения $u(t, x)$ задачи $(1.8),(1.9)$ является компактным множеством и имеет два непрерывных фронта (свободные поверхности), отделяющих область, где $u(t, x) \equiv 0$, от области, где $u(t, x)>0$ (см. [2], [14]-[17]):

$$
\begin{aligned}
& \eta_{+}(t)=\sup \{x: u(t, x)>0\}-\text { правый фронт, } \\
& \eta_{-}(t)=\inf \{x: u(t, x)>0\}-\text { левый фронт. }
\end{aligned}
$$

"Несимметричность" конвективного оператора приводит к тому, что $\eta_{-}(t)$ и $\eta_{+}(t)$ имеют различную асимптотику по $t$ : в частности, $\eta_{+}(t) \geqslant-\eta_{-}(t) \forall t>0$ при $\chi>0$ и $u_{0}(x)=u_{0}(-x)$ (см. [14]-[17]).

Характер поведения обоих фронтов решения задачи Коши для уравнения медленной диффузии со слабой конвекцией $(\lambda>(q+1) / 2)$ при $t \rightarrow \infty$ установлен в работе [16], а в [17] найдены главные члены их возможного асимптотического разложения. Начальная эволюция правого и левого фронтов обобщенных непрерывных решений задач Коши для уравнения медленной диффузии со слабой $(\lambda>(q+1) / 2)$ и "не очень" сильной $(q<\lambda<(q+1) / 2)$ конвекцией, а также левого фронта носителей решений этих задач для уравнений диффузии с "очень" сильной $(\lambda<1, \lambda<q)$ конвекцией были установлены в работах [14], [15]. В частности, в них были получены необходимые и достаточные условия на рост $u_{0}(x)$ в окрестности ее носителя, обеспечивающие нерасширение носителя решения в течение некоторого времени, называемого временем ожидания (эффект временной задержки носителя решения или ЗРН-эффект), а также оценки минимальной скорости расширения носителя в случае, если эти условия не выполнены. Для уравнений диффузии с "очень" сильной конвекцией установлено также условие на поведение $u_{0}(x)$ в окрестности $\eta_{-}(0)$, при выполнении которого стартовое движение левого фронта носителя происходит в противоположном диффузии направлении (обратное движение носителя). В работах [18], [19] найдены некоторые (неточные) оценки скорости движения обоих фронтов носителя решения уравнения (1.8) с сингулярной $u_{0}^{q}(x)=\delta(x)$.

Случаи, когда решения не обладают свойством конечности скорости распространения носителя, рассмотрены в [20]-[22]. В частности, в [21] показано, что оба фронта решения задачи Коши для уравнения быстрой диффузии со слабой и "не очень" сильной конвекцией обращаются в бесконечность при любых $t>0$, а в случае диффузии с "очень" сильной конвекцией - только правый фронт. В работе [22] найдены ограничения на поведение $u_{0}(x)$ при $x \rightarrow-\infty$, при которых левый фронт носителя решения уравнения диффузии с "очень" сильной конвекцией становится конечным за конечное время, даже если в начальный момент времени он находился на бесконечности (эффект мгновенной компактификации носителя или МКН-эффект). МКН-эффект для энергетических решений задачи Коши (1.1), (1.2) при $m=1$ был установлен в [23]. Гладкость фронтов решения уравнения медленной диффузии с конвекцией изучалась в [24].

Эволюция левого фронта носителя энергетического решения уравнения типа многомерной медленной диффузии с конвекцией без учета поведения $u_{0}(x)$ в окрестности ее носителя была исследована в работе [25]. Некоторые оценки сверху размеров носителей энергетических решений задачи $(1.1),(1.2)$ при $q<p, \lambda>0$ без учета локальных свойств $u_{0}(x)$ были получены в работах [26], [27], соответственно, для больших и малых значений времени. В случае $n=1$ многими авторами изучалось асимптотическое поведение при $t \rightarrow \infty$ решений полулинейных уравнений с конвективным членом (см., например, [28]-[30]).

Целью настоящей работы является исследование эволюции носителей энергетических решений (1.1)-(1.3) как при малых, так и при больших значениях времени. В частности, найдены близкие к точным условия на поведе- 
ние $u_{0}(x)$ в окрестности ее носителя, обеспечивающие возникновение ЗРНэффекта. В случае невыполнения этих условий установлены в определенном смысле точные оценки скорости расширения носителя, а также ограничения на показатели нелинейности уравнения, при которых эта скорость не зависит ни от начальной функции, ни от размерности $\Omega$. Для уравнений типа диффузии с "очень" сильной конвекцией найдены близкие к точным условия, обеспечивающие обратное стартовое движение левого фронта, а также установлены точные оценки скорости этого движения. Метод исследования эволюции носителей в работе основан на получении различных локальных энергетических оценок решений (оценок типа принципа Сен-Венана в теории упругости [31], [23], [27]) и не использует никаких вариантов барьерной техники, применимой только в уравнениях второго порядка.

\section{§ 2. Формулировка основных результатов}

ОПРЕДЕЛЕНИЕ 1. Энергетическим обобщенным решением $u(t, x)$ задачи (1.1)-(1.3) будем называть функцию

$$
\begin{aligned}
& u(t, x) \in V\left(G_{T}\right)=\left\{\begin{array}{ll}
v(t, x): & v \in L_{p+1}(0, T ; W) \cap L_{\lambda+1}((0, T) \times \Omega), \\
\left(|v|^{q-1} v\right)_{t} \in L_{\frac{p+1}{p}}\left(0, T ; W^{*}\right)+L_{\frac{\lambda+1}{\lambda}}((0, T) \times \Omega)
\end{array}\right\}, \\
& W(\Omega)=\left\{v \in W_{p+1}^{m}(\Omega) \cap L_{q+1}(\Omega):\left.D_{x}^{\alpha} v\right|_{\partial \Omega}=0,|\alpha| \leqslant m-1\right\},
\end{aligned}
$$

удовлетворяющую интегральному тождеству

$$
\begin{aligned}
\int_{0}^{T}\left\langle\left(|u|^{q-1} u\right)_{t}, w\right\rangle d t & +\int_{0}^{T}\langle\chi \cdot \nabla b(t, u), w\rangle d t \\
& +\sum_{|\alpha|=m} \int_{G_{T}} a_{\alpha}\left(t, x, u, D_{x} u, \ldots, D_{x}^{m} u\right) D_{x}^{\alpha} w d x d t=0
\end{aligned}
$$

для произвольной функции $w \in L_{p+1}\left(0, T ; W_{p+1}^{m}(\Omega)\right) \cap L_{\lambda+1}((0, T) \times \Omega)$, а также условию (1.2) в смысле пространства $C\left([0, T] ; L_{q+1}(\Omega)\right)$. Здесь $\langle u, w\rangle-$ спаривание элементов пространств $W$ и $W^{*}$.

ЗАМЕчАнИЕ 1 . В случае ограниченной области $\Omega, u_{0}(x) \in L_{\infty}(\Omega)$, при $m=1$, $q<p, \lambda \leqslant p+(p+1)(q+1) / n$ или при $m=1, \lambda<p \leqslant q$, а также при $m=1, \lambda \leqslant p(q+1) /(p+1), u_{0}(x) \in L_{q}(\Omega)$, существование и единственность энергетического решения задачи (1.1)-(1.3) следует из работы [13] и леммы 8.3 из дополнения.

Без ограничения общности, очевидно, можно считать, что

$$
\chi=\left(\chi_{1}, 0,0, \ldots, 0\right), \quad \chi_{1}=\text { const } \neq 0 .
$$

Введем теперь следующие семейства подобластей:

1) конусов раствора $\Lambda$ с вершиной в точке $\left(y_{1}+s, y^{\prime}\right) \in \mathbb{R}^{n}$

$$
\Omega^{(1)}(\Lambda, y, s)=\left\{x \in \Omega: x_{1}<s+y_{1}-\Lambda\left|x^{\prime}-y^{\prime}\right|\right\} \quad \forall s \in \mathbb{R}^{1}, \quad \Lambda>0 ;
$$

2) полупространств

$$
\begin{aligned}
& \Omega^{(2)}(y, \psi, s)=\{x \in \Omega:(\psi, x)<s+l\} \\
& \forall s \in \mathbb{R}^{1}, \quad y=l \psi, \quad l \in \mathbb{R}^{1}, \quad|\psi|=1, \quad \psi_{1} \geqslant 0, \quad \psi \in \mathbb{R}^{n} .
\end{aligned}
$$


По этим семействам определим соответственно семейства функций, характеризующих эволюцию носителя произвольного энергетического решения задачи (1.1)-(1.3):

$$
\begin{aligned}
& \eta^{(1)}(\Lambda, y, t)=\sup \left\{s: \operatorname{supp} u(t, \cdot) \cap \Omega^{(1)}(\Lambda, y, s)=\varnothing\right\} \\
& \eta^{(2)}(y, \psi, t)=\sup \left\{s: \operatorname{supp} u(t, \cdot) \cap \Omega^{(2)}(y, \psi, s)=\varnothing\right\}
\end{aligned}
$$

При этом, учитывая определение правого и левого фронтов из (1.10), в случае $\chi_{1}>0$ функцию $\eta^{(1)}(\Lambda, y, t)\left(\eta^{(2)}(y, \psi, t)\right)$ будем обозначать $\eta_{-}^{(1)}(\Lambda, y, t)$ $\left(\eta_{-}^{(2)}(y, \psi, t)\right)$ и говорить об эволюции левого фронта носителя; соответственно при $\chi_{1}<0$ функцию $\eta^{(1)}(\Lambda, y, t)\left(\eta^{(2)}(y, \psi, t)\right)$ будем обозначать $\eta_{+}^{(1)}(\Lambda, y, t)$ $\left(\eta_{+}^{(2)}(y, \psi, t)\right)$ и говорить об эволюции правого фронта.

ОПредЕлЕниЕ 2. Энергетическое решение $u(t, x)$ задачи (1.1)-(1.3) обладает свойством конечности скорости распространения возмущений (КСРсвойством), если существует $\tau>0$ такое, что выполнено $\left|\eta^{(1)}(\Lambda, y, t)\right|<\infty$ $\left(\left|\eta^{(2)}(y, \psi, t)\right|<\infty\right) \forall t, 0<t<\tau$.

ОПРЕДЕЛЕНИЕ 3. Будем говорить, что имеет место задержка распространения носителя (ЗРН-эффект) решения $u(t, x)$, если существует $T^{*}>0$ такое, что $\eta^{(1)}(\Lambda, y, t) \geqslant \eta^{(1)}(\Lambda, y, 0)\left(\eta^{(2)}(y, \psi, t) \geqslant \eta^{(2)}(y, \psi, 0)\right)$ при любых $0<t \leqslant T^{*}$.

ОПРЕДЕЛЕНИЕ 4. Некоторую неубывающую неотрицательную непрерывную функцию $h(s)$ назовем правилъно монотонной, если для произвольного $\delta_{1}$, $0<\delta_{1}<\delta_{0} \leqslant 1$, существует $0<\delta_{2}=\delta_{2}\left(\delta_{1}\right)<1$ такое, что $h\left(\left(1-\delta_{1}\right) s\right) \geqslant \delta_{2} h(s)$ $\forall s>0$.

Введем теперь семейства функций, характеризующие поведение начальной функции $u_{0}(x)$ в окрестности ее носителя:

$h_{0}^{(1)}(\Lambda, y, s)=\int_{\Omega^{(1)}(\Lambda, y, s)}\left|u_{0}(x)\right|^{q+1} d x, \quad h_{0}^{(2)}(y, \psi, s)=\int_{\Omega^{(2)}(y, \psi, s)}\left|u_{0}(x)\right|^{q+1} d x$.

Обозначим через $h^{(1)}(\Lambda, y, s)$ и $h^{(2)}(y, \psi, s)$ :

$$
\begin{aligned}
& 0 \leqslant h_{0}^{(1)}(\Lambda, y, s) \leqslant h^{(1)}(\Lambda, y, s)<\infty \\
& 0 \leqslant h_{0}^{(2)}(y, \psi, s) \leqslant h^{(2)}(y, \psi, s)<\infty
\end{aligned}
$$

минимальные правильно монотонные мажоранты соответственно функций $h_{0}^{(1)}(\Lambda, y, s)$ и $h_{0}^{(2)}(y, \psi, s)$.

Введем также следующие множества параметров $(\Lambda, y)$ и $(y, \psi)$ :

$$
\begin{gathered}
V_{1}=\left\{(\Lambda, y): \operatorname{supp} u_{0}(x) \cap \Omega^{(1)}\left(\Lambda^{\prime}, y, 0\right)=\varnothing,\right. \\
\left.\operatorname{supp} u_{0}(x) \cap \overline{\Omega^{(1)}}(\Lambda, y, 0) \neq \varnothing\right\} \quad \forall \Lambda^{\prime} \in(0, \Lambda), \\
V_{2}=\left\{(y, \psi): \operatorname{supp} u_{0}(x) \cap \Omega^{(2)}(y, \psi, 0)=\varnothing,\right. \\
\left.\operatorname{supp} u_{0}(x) \cap \overline{\Omega^{(2)}}(y, \psi, 0) \neq \varnothing\right\} .
\end{gathered}
$$


Из определения множеств $V_{1}$ и $V_{2}$, очевидно, вытекает, что

$$
\begin{aligned}
(\Lambda, y) \in V_{1} & \Rightarrow \operatorname{supp} u_{0}(x) \subseteq \mathbb{R}^{n} \backslash \Omega^{(1)}(\Lambda, y, 0) \Rightarrow \eta^{(1)}(\Lambda, y, 0)=0 \\
& \Rightarrow h^{(1)}(\Lambda, y, 0)=0, \\
(y, \psi) \in V_{2} & \Rightarrow \operatorname{supp} u_{0}(x) \subseteq \mathbb{R}^{n} \backslash \Omega^{(2)}(y, \psi, 0) \Rightarrow \eta^{(2)}(y, \psi, 0)=0 \\
& \Rightarrow h^{(2)}(y, \psi, 0)=0 .
\end{aligned}
$$

В дальнейшем будем предполагать, что $(\Lambda, y) \in V_{1},(y, \psi) \in V_{2}$, а параметры $(\Lambda, y),(y, \psi)$ и индексы ${ }^{(1)},{ }^{(2)}$ в формулировках, справедливых для $\eta^{(1)}(\Lambda, y, t)$ и $\eta^{(2)}(y, \psi, t)$, для краткости будем опускать.

\section{1. Прямое движение левого фронта.}

Теорема 1. Пусть параметры уравнения (1.1) удовлетворяют соотношениям $\chi_{1}>0, q<p<\lambda$. Зафиксируем произвольно постоянную $\delta_{3}, 0<\delta_{3}<\infty$, и определим функиию

$$
v_{1}(t)=\inf \left\{s:\left(\operatorname{th}(s)^{\frac{p-q}{q+1}}\right)^{\beta_{1}}<\delta_{3} s\right\} \quad \forall t>0 .
$$

Тогда произвольное энергетическое решение $и(t, x)$ задачи (1.1)-(1.3) обладает КСР-свойством и для левого фронта носителя верна оченка:

$$
\eta_{-}(t) \geqslant a_{1} \max \left\{\eta^{(d)}(t), \eta_{-}^{(c)}(t)\right\} \quad \forall t>0, \quad a_{1}=a_{1}\left(\delta_{3}\right)=\text { const }>0,
$$

¿de

$$
\begin{aligned}
& \eta^{(d)}(t)=-\left(\operatorname{th}\left(v_{1}(t)\right)^{\frac{p-q}{q+1}}\right)^{\beta_{1}}, \quad \beta_{1}=\frac{q+1}{m(p+1)(q+1)+n(p-q)}, \\
& \eta_{-}^{(c)}(t)= \begin{cases}-\chi_{1}^{-\frac{p-q}{\lambda-p} \beta_{2}} t^{\beta_{2}}, & h_{0}(s)=h_{0}^{(1)}(\Lambda, y, s), \quad \beta_{2}=\frac{\lambda-p}{m(p+1)(\lambda-q)-p+q}, \\
-\infty, & h_{0}(s)=h_{0}^{(2)}(y, \psi, s) .\end{cases}
\end{aligned}
$$

ПримеР 1. Пусть начальная функция $u_{0}(x)$ такова, что соответствующая функция $h_{0}(s)$ удовлетворяет неравенству

$$
h_{0}(s) \leqslant h(s) \equiv k_{1} s^{\alpha}, \quad k_{1}>0, \quad 0<\alpha \leqslant \alpha_{1} \equiv n+\frac{m(p+1)(q+1)}{p-q} .
$$

Тогда, очевидно,

$$
v_{1}(t)=\left(\delta_{3}^{-\beta_{1}^{-1}} k_{1}^{\frac{p-q}{q+1}}\right)^{\beta(\alpha)} t^{\beta(\alpha)}, \quad \beta(\alpha)=\frac{q+1}{m(p+1)(q+1)+(n-\alpha)(p-q)}
$$

и, следовательно,

$$
\eta^{(d)}(t)=-\delta_{3} v_{1}(t) \quad \forall t>0
$$

ЗАмЕчАниЕ 2. Очевидно, что функция $v_{1}(t)$ из $(2.7)$, строящаяся по $h(s)$ из примера 1 , при $\alpha=\alpha_{1}$ тождественно равна нулю на некотором интервале $\left(0, T^{*}\right), T^{*}>0$.

ЗАмечание 3 . Легко проверить, что $\beta(\alpha)$ из (2.12) и $\beta_{2}$ из (2.10) удовлетворяют неравенству $\beta(\alpha) \geqslant \beta_{2}$ при следующих соотношениях на параметры: 

a) $0 \leqslant \alpha<\alpha_{1}, \lambda \leqslant \lambda_{c r_{1}} \equiv p+\frac{(m(p+1)-1)(q+1)}{n}$ либо
b) $\alpha_{2} \equiv n-\frac{(m(p+1)-1)(q+1)}{\lambda-p} \leqslant \alpha<\alpha_{1}, \lambda>\lambda_{c r_{1}}$.

Пример 1 и замечания 2, 3 легко приводят к следующим следствиям из теоремы 1.

СлЕдСтвиЕ 1 (стартовое распространение носителя).

1. Если $u_{0}(x)$ удовлетворяет следующему условию малости:

$$
h_{0}(s) \leqslant k s^{\alpha_{1}} \quad \forall s, 0 \leqslant s<s_{0}, \quad k<\infty, \quad \alpha_{1}=n+\frac{m(p+1)(q+1)}{p-q},
$$

то имеет место ЗРН-эффект.

2. Если же условие (2.14) не выполняется, т.е.

$$
\lim _{s \rightarrow 0} h_{0}(s) s^{-\alpha_{1}}=\infty
$$

то возможны следующие варианты:

а) $n p u \lambda \leqslant \lambda_{\text {cr }_{1}}=p+\frac{(m(p+1)-1)(q+1)}{n}$ cуществует $0<T^{*}<\infty$ такое, что оценка (2.8) эквивалентна оценке

$$
\eta_{-}(t) \geqslant a_{2} \eta^{(d)}(t) \quad \forall t<T^{*}, \quad a_{2}=a_{2}\left(\delta_{3}\right)=\text { const }>0 ;
$$

b) при $\lambda>\lambda_{\text {сr } 1}$ имеет место следующая универсальная оценка, не зависящая от начальной функции $u_{0}(x)$ :

$$
\eta_{-}(t) \geqslant a_{3} \eta_{-}^{(c)}(t) \quad \forall t<T^{*}, \quad a_{3}=\text { const }>0 ;
$$

если к тому же дополнительно выполнено следующее условие малости для $u_{0}(x)$ :

$$
\begin{gathered}
h_{0}(s) \leqslant k_{1} s^{\alpha_{2}} \\
\forall s, 0 \leqslant s<s_{0}<\infty, \quad k<\infty, \quad \alpha_{2}=n-\frac{(m(p+1)-1)(q+1)}{\lambda-p},
\end{gathered}
$$

то оценка (2.8) эквивалентна (2.16).

СледСТвиЕ 2 (эволюция носителя при $t \rightarrow \infty$ ). Пусть $\lambda \geqslant \lambda_{c r_{1}}$. Тогда оценка (2.8) эквивалентна оценке

$$
\eta_{-}(t) \geqslant-A_{1} t^{\beta_{1}} \quad \forall t>T^{*}>0, \quad A_{1}=\text { const }>0, \quad \beta_{1}-\text { из }(2.9) .
$$

Если же $\lambda<\lambda_{\text {cr }_{1}}$, то оценка (2.8) эквивалентна оценке

$$
\eta_{-}(t) \geqslant a_{3} \eta_{-}^{(c)}(t) \quad \forall t>T^{*}, \quad a_{3}=\text { const }>0 .
$$

Теорема 2. Пустъ параметры уравнения (1.1) удовлетворяют соотношениям $q<\lambda=p, \chi_{1}>0$. Тогда произвольное энергетическое решение задачи (1.1)-(1.3) обладает KCP-свойством и существует $0<T^{*}\left(\psi_{1}\right)<\infty \quad \forall \psi_{1}>0$ такое, что для левого фронта носителя верна оценка (2.16) при $0<t<T^{*}$, а также выполнено

$$
\eta_{-}(t) \geqslant-A_{2}\left(\psi_{1} \chi_{1}\right) \ln t \quad \forall t \geqslant T^{*}, \quad A_{2}=A_{2}\left(\psi_{1} \chi_{1}\right)=\text { const }>0 .
$$


ТЕОРема 3. Пусть параметры уравнения (1.1) удовлетворяют соотношениям $\chi_{1}>0, q<p, \lambda \leqslant p$. Зафиксируем произвольно постоянную $\delta_{4}>0 u$ определим число $s_{1}=s_{1}\left(\psi_{1} \chi_{1}\right) \geqslant 0$ - максимальный корень уравнения

$$
\begin{aligned}
& \left(\psi_{1} \chi_{1}\right)^{(\nu-1)(1+\mu) \beta_{3}}\left(s^{1-\nu} h(s)^{1+\mu}\right)^{\mu \beta_{3}} \\
& \quad+s^{\beta_{4}}\left(\psi_{1} \chi_{1}\right)^{(\nu-1)(1+\mu)\left(1-\beta_{4}\right) \beta_{3}}\left(s^{1-\nu} h(s)^{1+\mu}\right)^{\mu \beta_{3}\left(1-\beta_{4}\right)}=\delta_{4} s,
\end{aligned}
$$

где

$$
\begin{aligned}
& \beta_{3}=(m(p+1)(1+\mu)-1+\nu)^{-1}, \quad \beta_{4}=(1-\nu)(m(p+1)(1+\mu))^{-1}, \\
& \nu=n(p-\lambda)[m(p+1)(q+1)+n(p-\lambda)]^{-1}, \quad \mu=m n^{-1}(p+1) \nu .
\end{aligned}
$$

Тогда произвольное энергетическое решение и(t, $x)$ задачи (1.1)-(1.3) обладает КСР-свойством и для левого фронта верна оценка

$$
\eta_{-}(t) \geqslant a_{4} \max \left\{\eta_{-}^{(d)}(t), \eta_{-}^{(s c)}\left(\psi_{1} \chi_{1}\right)\right\} \quad \forall t>0, \quad a_{4}=a_{4}\left(\delta_{3}, \delta_{4}\right)=\text { const }>0,
$$

где $\eta^{(d)}(t), \delta_{3}>0$ - из теоремь $1, \eta_{-}^{(s c)}\left(\psi_{1} \chi_{1}\right)=-\delta_{4} s_{1}\left(\psi_{1} \chi_{1}\right)$. Kроме того, существует $k_{2}\left(\psi_{1} \chi_{1}\right)>0$ такое, что при выполнении условия малости

$$
h_{0}(s) \leqslant k_{2} s^{\alpha_{2}} \quad \forall s, 0 \leqslant s<s_{0} \leqslant \infty, \quad \alpha_{2}=n+\frac{(m(p+1)-1)(q+1)}{p-\lambda}
$$

имеет место ЗРН-эффект с $T^{*}=T^{*}\left(s_{0}, \psi_{1} \chi_{1}\right)$, причем $T^{*}\left(s_{0}, \psi_{1} \chi_{1}\right) \rightarrow \infty$ при $s_{0} \rightarrow \infty$.

ЗАмечАниЕ 4 . Нетрудно проверить, что неравенство $\alpha_{2} \leqslant \alpha_{1}$ эквивалентно неравенству $\lambda \leqslant \lambda_{c r_{2}}=q+(p-q)(m(p+1))^{-1}$.

Из теорем 1-3 и замечания 4, очевидно, вытекает

СлеДСтвиЕ 3. Пусть в (1.1) дополнительно выполнено $\lambda>\lambda_{\mathrm{cr}_{2}}$. Тогда если $u_{0}(x)$ удовлетворяет условию малости (2.14), то имеет место ЗРН-эффект. Если же $\lambda \leqslant \lambda_{\text {rr }_{2}}$, то для возникновения ЗРН-эффекта достаточно выполнения более слабого, чем (2.14), условия (2.25).

ЗАмечание 5. При $n=m=p=1, \lambda_{c r_{2}}<\lambda<\lambda_{c r_{1}}, t \rightarrow \infty$ оценки (2.8), (2.21), (2.24) соответствуют результатам работы [17].

ТЕОРема 4. Пусть уравнение (1.1) является уравнением типа быстрой дифбузии с “очень сильной” конвекцией:

$$
\chi_{1}>0, \quad \lambda<p \leqslant q, \quad p \geqslant \frac{n q-m(q+1)}{n+m(q+1)} .
$$

Тогда произвольное энергетическое решение рассматриваемой задачи обладает конечным левым фронтом и имеет место следующая оченка:

$$
\eta_{-}(t) \geqslant a_{5} \max \left\{\eta_{-}^{(c)}(t), \eta_{-}^{(s c)}\left(\psi_{1} \chi_{1}\right)\right\} \quad \forall t>0, \quad a_{5}=a_{5}\left(\delta_{4}\right)=\text { const }>0
$$

где $\eta_{-}^{(c)}(t), \eta_{-}^{(s c)}\left(\psi_{1} \chi_{1}\right)$ определяются равенствами (2.10) и (2.24) соответственно. Кроме того, при выполнении условия малости (2.25) имеет место ЗРН-эббект с $0<T^{*}=T^{*}\left(s_{0}, \psi_{1} \chi_{1}\right)$, причем $\lim _{s_{0} \rightarrow \infty} T^{*}\left(s_{0}, \psi_{1} \chi_{1}\right)=\infty$.

СлЕДСТВИЕ 4. Из оценок (2.24), (2.27) легко получить следующее соотношение:

$$
\eta_{-}(t) \geqslant-\widetilde{a}_{5}\left(\chi_{1}\right) \psi_{1}^{-\frac{1-\nu}{m(p+1)-1+\nu}} \quad \forall t>0, \quad \widetilde{a}_{5}\left(\chi_{1}\right)=\text { const }>0 .
$$




\section{2. Обратное движение левого фронта носителя.}

Теорема 5. Пусть параметры уравнения (1.1) удовлетворяют соотношениям

$$
\chi_{1}>0, \quad \lambda<p, \quad \lambda<q, \quad p \geqslant \frac{n q-m(q+1)}{n+m(q+1)} .
$$

Тогда существует положительная постоянная $k_{3}<k_{2}\left(k_{2}-\right.$ из теоремъ 3$)$ такая, что при выполнении условия малости

$$
h_{0}(s) \leqslant k_{3} s^{\alpha_{2}} \quad \forall 0 \leqslant s<s_{0} \leqslant \infty
$$

для левого фронта носителя произвольного энергетического решения выполнено

$$
\begin{gathered}
\eta_{-}(t) \geqslant a_{5} f^{-1}\left(a_{6}\left(\psi_{1} \chi_{1}\right)^{1-\nu_{1}} t\right) \\
\forall t<T^{*}\left(s_{0}, \psi_{1} \chi_{1}\right), \quad a_{5}=\text { const }>0, \quad a_{6}=\text { const }>0,
\end{gathered}
$$

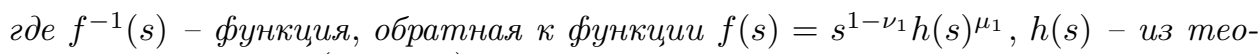
реми $1, \lim _{s_{0} \rightarrow \infty} T^{*}\left(s_{0}, \psi_{1} \chi_{1}\right)=\infty$,

$$
\nu_{1}=n(q-\lambda)(m(p+1)(q+1)+n(p-\lambda))^{-1}, \quad \mu_{1}=m n^{-1}(p+1) \nu_{1} .
$$

Также существует время $T^{* *}\left(s_{1}\left(\psi_{1} \chi_{1}\right)\right) \geqslant T^{*}\left(s_{0}, \psi_{1} \chi_{1}\right)\left(s_{1}\left(\psi_{1} \chi_{1}\right)-\right.$ из $\left.(2.22)\right)$ такое, что

$$
\begin{gathered}
\eta_{-}(t) \geqslant A_{3} \psi_{1} \chi_{1} t^{\beta_{5}} \quad \forall t>T^{* *}, \quad A_{3}=\text { const }>0, \\
\beta_{5}=\frac{m(p+1)(q+1)+n(p-\lambda)}{m(p+1)(q+1)+n(p-q)} .
\end{gathered}
$$

ЗАмЕчАниЕ 6. Последнее ограничение в (2.26) и (2.28) имеет "техническую" природу (обеспечивает соответствующее вложение) и не встречается в работах [14], [15], основанных на барьерной технике.

\section{3. Движение правого фронта носителя.}

ТЕОрема 6. Пусть параметры уравнения (1.1) удовлетворяют соотношениям

$$
q<\lambda<p+m(p+1)(q+1) n^{-1}, \quad q<p, \quad \chi_{1}<0 .
$$

Зафиксируем произвольно постоянную $0<\delta_{5}<\infty$ и определим функиию

$$
\begin{gathered}
v_{2}(t)=\inf \left\{s:-\psi_{1} \chi_{1} t^{\beta_{5}} h(s)^{\beta_{6}}<\delta_{5} s\right\} \quad \forall t>0, \\
\beta_{6}=\frac{m(p+1)(\lambda-q)}{m(p+1)(q+1)+n(p-q)},
\end{gathered}
$$

$\beta_{5}-$ из (2.32), $h(s)$ - из теоремы 1. Тогда произвольное энергетическое решение задачи (1.1)-(1.3) обладает КСР-свойством и для правого фронта носителя решения имеет место оценка

$$
\eta_{+}(t) \geqslant a_{7} \min \left\{\eta^{(d)}(t), \eta_{+}^{(c)}(t)\right\} \quad \forall t>0, \quad a_{7}=a_{7}\left(\delta_{3}, \delta_{5}\right)=\text { const }>0,
$$

где $\eta_{+}^{(c)}=\psi_{1} \chi_{1} t^{\beta_{5}} h\left(v_{2}(t)\right)^{\beta_{6}}, \eta^{(d)}(t) u \delta_{3}>0-u з$ теоремъ 1. 
ПримеР 2. Пусть начальная функция $u_{0}(x)$ такова, что соответствующая ей функция $h_{0}(s)$ удовлетворяет следующему условию:

$$
h_{0}(s) \leqslant h(s)=k s^{\alpha}, \quad 0<\alpha<\alpha_{3}=\frac{n(p-q)}{m(p+1)(\lambda-q)}+\frac{q+1}{\lambda-q}, \quad k<\infty .
$$

Тогда, очевидно,

$$
\begin{gathered}
v_{2}(t)=\left(-\psi_{1} \chi_{1} \delta_{5}^{-1} k^{\beta_{6}}\right)^{\left(1-\alpha \beta_{6}\right)^{-1}} t^{\widetilde{\beta}(\alpha)}, \\
\widetilde{\beta}(\alpha)=\frac{m(p+1)(q+1)+n(p-\lambda)}{m(p+1)(q+1-\alpha(\lambda-q))+n(p-q)},
\end{gathered}
$$

и, следовательно, $\eta_{+}^{(c)}(t)=-\delta_{5} v_{2}(t) \forall t, 0<t<T^{*}$.

ЗАМЕчАНИЕ 7 . Очевидно, что функция $v_{2}(t)$ из $(2.33)$, строящаяся по $h(s)$ из примера 2 , при $\alpha=\alpha_{3}$ тождественно равна нулю на некотором интервале $\left(0, T^{*}\right), T^{*}>0$.

ЗАмЕчАНИЕ 8. Нетрудно проверить, что $\beta(\alpha)$ из $(2.12)$ и $\widetilde{\beta}(\alpha)$ из $(2.36)$ удовлетворяют неравенствам $\beta(\alpha) \geqslant \widetilde{\beta}(\alpha), 0 \leqslant \alpha<\min \left\{\alpha_{1}, \alpha_{3}\right\}$ при следующих соотношениях параметров:

a) $\alpha_{2}<\alpha<\alpha_{3}, \lambda<\lambda_{c r_{2}}=q+\frac{p-q}{m(p+1)}, \alpha_{2}-$ из (2.18), $\alpha_{3}-$ из (2.35), или

b) $0 \leqslant \alpha<\alpha_{2}, \quad \lambda>\lambda_{c r_{1}}=p+(m(p+1)-1)(q+1) n^{-1}$.

Примеры 1, 2 и замечания 2, 7, 8 позволяют получить следующие следствия из теоремы 6.

СлЕДСтвиЕ 5 (стартовое движение носителя). При

$$
\lambda>\lambda_{c r_{1}}=p+\frac{(m(p+1)-1)(q+1)}{n}
$$

существует $0<T^{*}<\infty$ такое, что оценка (2.34) эквивалентна оценке

$$
\eta_{+}(t) \geqslant a_{8} \eta_{+}^{(c)}(t) \quad \forall t<T^{*}, \quad a_{8}=\text { const }>0 .
$$

Если к тому же $u_{0}(x)$ удовлетворяет условию малости (2.18), то (2.34) эквивалентна оценке

$$
\eta_{+}(t) \geqslant a_{9} \eta^{(d)}(t) \quad \forall t<T^{*}, \quad a_{9}=\text { const }>0 .
$$

При выполнении более сильного условия малости (2.14) имеет место ЗРНэбфект.

При $\lambda_{c r_{2}} \leqslant \lambda \leqslant \lambda_{c r_{1}}$ оценка (2.34) эквивалентна соотношению (2.38) при всех $t<T^{*}$. Если $u_{0}(x)$ удовлетворяет условию малости (2.14), то имеет место ЗРН-эфбект.

При $\lambda<\lambda_{\text {сr }_{2}}$ оценка (2.34) эквивалентна (2.38). При выполнении для $u_{0}(x)$ условия малости (2.18) оценка (2.34) эквивалентна (2.37). Если же $u_{0}(x)$ удовлетворяет более сильному условию малости

$$
h_{0}(s) \leqslant k_{4} s^{\alpha_{3}} \quad \forall s, 0<s<s_{0}<\infty, \quad k_{4}<\infty,
$$

то имеет место ЗРН-эфбект с $T^{*}=T^{*}\left(k_{4}, \psi_{1} \chi_{1}\right)<\infty$. 
СлЕДСТвиЕ 6 (эволюция носителя при $t \rightarrow \infty$ ). При $\lambda \leqslant \lambda_{c r_{1}}$ для правого фронта носителя решения имеет место оценка

$$
\eta_{+}(t) \geqslant A_{4} \psi_{1} \chi_{1} t^{\beta_{5}} \quad \forall t, t>T^{*}>0, \quad A_{4}=\text { const }>0, \quad \beta_{5}-u 3(2.32) .
$$

Если же $\lambda>\lambda_{c r_{1}}$, mo

$$
\eta_{+}(t) \geqslant-A_{5} t^{\beta_{1}} \quad \forall t, t>T^{*}>0, \quad A_{5}=\text { const }>0, \quad \beta_{1}-u 3(2.9) .
$$

ЗАмечАнИЕ 9. Пусть в (1.1) $m=n=p=1, \lambda>\lambda_{c r_{2}}=2^{-1}(q+1), q<1$, $u_{0}(x) \in C\left(\mathbb{R}^{1}\right)$. Тогда для выполнения условия малости $(2.14)$, а следовательно, и для возникновения ЗРН-эффекта достаточным условием является:

$$
0 \leqslant u_{0}(x) \leqslant k x^{\frac{2}{1-q}} \quad \forall x, 0 \leqslant x<x_{0}, \quad k<\infty,
$$

что соответствует результатам, установленным в [14], [15].

ЗАмечАнИЕ 10. Пусть в уравнении (1.1) $m=n=p=1, u_{0}(x) \in C\left(\mathbb{R}^{1}\right)$, $\lambda \leqslant \lambda_{c r_{2}}=2^{-1}(q+1), q<1, \chi_{1}>0$ или $\lambda<1 \leqslant q, \chi_{1}>0$. Тогда для выполнения условия малости (2.25), обеспечивающего возникновение ЗРН-эффекта, достаточным является условие:

$$
0 \leqslant u_{0}(x)<\widetilde{k} x^{\frac{1}{1-\lambda}} \quad \forall x, 0 \leqslant x<x_{0}<\infty, \quad \widetilde{k}<\left(k_{2} \alpha_{2}\right)^{\frac{1}{q+1}}, \quad \alpha_{2}=1+\frac{q+1}{1-\lambda},
$$

где $k_{2}>0$ - из теоремы 3. Это соответствует результатам работ [14], [15].

ЗАмЕчАНИЕ 11. Пусть в условиях замечания 10 для $u_{0}(x)$ выполнено следующее:

$$
u_{0}(x)=\widetilde{k} x^{\frac{1}{1-\lambda}} \quad \forall x, 0<x<x_{0}<\infty .
$$

Тогда, очевидно, $h(s)=\alpha_{2}^{-1} \widetilde{k}^{q+1} s^{\alpha_{2}}$ и если $\widetilde{k}>\left(\alpha_{2} k_{2}\right)^{1 /(q+1)}\left(k_{2}-\right.$ из теоремы 3$)$, то соотношения (2.24) и (2.27) эквивалентны оценке

$$
\eta_{-}(t) \geqslant-a_{10} t^{\frac{1-\lambda}{q+1-2 \lambda}} \quad \forall t<T^{*}<\infty, \quad a_{10}=\text { const }>0 .
$$

Если же в $(2.42)$ выполнено $\widetilde{k}<\left(k_{3} \alpha_{2}\right)^{1 /(q+1)}\left(k_{3}-\right.$ из теоремы 5$)$, то оценка (2.30) эквивалентна оценке

$$
\eta_{-}(t) \geqslant a_{11} t^{\frac{1-\lambda}{q+1-2 \lambda}} \quad \forall t<T^{*}<\infty, \quad a_{11}=\text { const }>0,
$$

что также соответствует результатам работ [14], [15].

ЗАмечАниЕ 12 . Пусть в (1.1) $q=\lambda<p, b(t, u) \equiv|u|^{q-1} u$. Тогда, сделав замену переменных $u(t, x)=v\left(t, x_{1}-\chi_{1} t, x^{\prime}\right)$, легко проверить, что функция $v(t, x)$ удовлетворяет уравнению (1.1) с $b(t, v) \equiv 0$, следовательно, для левого (правого) фронта носителя решения $v(t, x)$ в силу теоремы $1 \widetilde{\eta}_{ \pm}(t) \geqslant-a_{12} \eta^{(d)}(t)$ $\forall t>0$. Возвращаясь к исходным переменным, получаем оценку

$$
\eta_{ \pm}(t) \geqslant \mp\left|\psi_{1} \chi_{1}\right| t-a_{12} \eta^{(d)}(t) \quad \forall t>0, \quad a_{12}=\text { const }>0 .
$$

Отсюда, очевидно, следует, что $\eta_{+}(t)>0 \forall t>0$. 
2.4. Геометрия носителей энергетических решений. Из (2.1)-(2.6), очевидно, вытекает, что для носителя произвольного энергетического решения $u(t, x)$ задачи (1.1)-(1.3) имеет место включение $\forall t>0$

$\operatorname{supp} u(t, \cdot) \subseteq \mathbb{R}^{n} \backslash\left[\bigcup_{(\Lambda, y) \in V_{1},(y, \psi) \in V_{2}}\left(\Omega^{(1)}\left(\Lambda, y, \eta^{(1)}(\Lambda, y, t)\right) \cup \Omega^{(2)}\left(y, \psi, \eta^{(2)}(y, \psi, t)\right)\right)\right]$.

Теорема 7. Пусть $и(t, x)$ - произвольное энергетическое решение задачи (1.1)-(1.3) при $\chi_{1}=(1,0, \ldots, 0), \operatorname{supp} u_{0} \subseteq\left\{x \in \mathbb{R}^{n}:|x| \leqslant R\right\}, R>0$. Тогда справедливы следующие утвержсения:

1) если в (1.1) дополнительно выполнено условие $q<\lambda<p$, то для носителя решения верно включение $\operatorname{supp} u(t, \cdot) \subseteq D^{(1)} \cap D_{t}^{(2)} \forall t>0$,

$$
\begin{aligned}
D^{(1)} & =\left\{x \in \Omega: x_{1} \geqslant-R-A_{6}+A_{7}\left|x^{\prime}\right|^{\beta_{7}}\right\}, \\
\beta_{7} & =m(p+1)+n(p-\lambda)(q+1)^{-1}, \\
D_{t}^{(2)} & =\left\{x \in \Omega: x_{1} \leqslant A_{8} t^{\beta_{5}}+\left[\left(R+A_{9} t^{\beta_{1}}\right)^{2}-\left|x^{\prime}\right|^{2}\right]^{\frac{1}{2}}\right\},
\end{aligned}
$$

$\beta_{1}, \beta_{5}-$ из (2.7) и (2.32) соответственно;

2) пусть в (1.1) $q<p, b(t, u) \equiv|u|^{q-1} u$; тогдa $\forall t>0$

$$
\operatorname{supp} u(t, \cdot) \subseteq D_{t}^{(3)}=\left\{x \in \Omega:\left(x_{1}-t\right)^{2}+\left|x^{\prime}\right|^{2} \leqslant\left(R+A_{10} t^{\beta_{1}}\right)^{2}\right\} \subseteq D^{(1)}
$$

3) в случае $\lambda<q<$ р существует $T^{*}>0$ mакое, что

$$
\operatorname{supp} u(t, \cdot) \subseteq D_{t}^{(4)} \cap D_{t}^{(5)} \subseteq D^{(1)} \quad \forall t>T^{*},
$$

где $D_{t}^{(4)}=\left\{x \in \Omega: x_{1} \geqslant-R+A_{11} t^{\beta_{5}}\right\}, D_{t}^{(5)}=\left\{x \in \Omega:\left|x^{\prime}\right| \leqslant R+A_{12} t^{\beta_{1}}\right\} ;$

4) если в (1.1) $q<p<\lambda$, то

$$
\operatorname{supp} u(t, \cdot) \subseteq D_{t}^{(2)} \cap D_{t}^{(6)} \quad \forall t>0
$$

$D_{t}^{(6)}=\left\{x \in \Omega: x_{1} \geqslant-R-A_{13} t^{\beta_{8}}\right\}, \beta_{8}=\beta_{1} \beta_{2}\left(\beta_{1}+\beta_{2}\right)^{-1}, \beta_{2}-u з(2.10) ;$

5) в случае $\lambda<p<q, p \geqslant \frac{n q-m(q+1)}{n+m(q+1)}$ cуществует $T^{*}>0$ такое, что

$$
\operatorname{supp} u(t, \cdot) \subseteq D^{(1)} \cap D_{t}^{(4)} \quad \forall t>T^{*} ;
$$

6) при $q<\lambda=$ существует $T^{*}>0$ maкое, что

$\operatorname{supp} u(t, \cdot) \subseteq D_{t}^{(2)} \cap D_{t}^{(7)} \quad \forall t>T^{*}, \quad D_{t}^{(7)}=\left\{x \in \Omega: x_{1} \geqslant-R-A_{14} \ln t\right\} ;$

7) если же $\lambda<p=q$, то

$$
\begin{aligned}
& \operatorname{supp} u(t, \cdot) \subseteq D_{t}^{(8)}=\left\{x \in \Omega: x_{1} \geqslant A_{15} t^{\beta_{5}}-\left[\left(R+A_{16} t^{\beta_{1}}\right)^{2}-\left|x^{\prime}\right|^{2}\right]^{\frac{1}{2}}\right\} \subseteq D^{(1)} \\
& \forall t>0,0<A_{i}<\infty, i=\overline{6,16} .
\end{aligned}
$$




\section{§ 3. Вспомогательные построения и утверждения}

По семейству областей $\Omega(s)$ из (2.1) и (2.2) определим следующие семейства подобластей:

$$
\begin{gathered}
K(s, \delta)=\Omega(s) \backslash \Omega(s-\delta) \quad \forall s \in \mathbb{R}^{1}, \quad \forall \delta>0, \\
G_{t_{1}}^{t_{2}}(s)=\left(t_{1}, t_{2}\right) \times \Omega(s), \quad Q_{t_{1}}^{t_{2}}(s, \delta)=\left(t_{1}, t_{2}\right) \times K(s, \delta) .
\end{gathered}
$$

В дальнейшем нами систематически будет использоваться интерполяционное неравенство Гальярдо-Ниренберга, которое в случае областей $K(s, \delta)$ примет вид

$$
\left\|D_{x}^{j} v\right\|_{a, K(s, \delta)} \leqslant d_{4} \delta^{-j-\frac{n(a-d)}{a d}}\|v\|_{d, K(s, \delta)}+d_{5}\left\|D_{x}^{m} v\right\|_{b, K(s, \delta)}^{\theta}\|v\|_{d, K(s, \delta)}^{1-\theta},
$$

$0 \leqslant|j|<m$. Здесь $v(x)-$ произвольная функция из пространства

$$
W_{b}^{m}(K(s, \delta)) \cap L_{d}(K(s, \delta)), \quad\|v\|_{a, B}^{a}=\int_{B}|v|^{a} d x,
$$

где $d>0, a>1, b>1, \theta \in[j / m, 1]$ определяется из равенства

$$
a^{-1}-j n^{-1}=\left(b^{-1}-m n^{-1}\right) \theta+(1-\theta) d^{-1},
$$

а постоянные $d_{4}<\infty, d_{5}<\infty$ зависят только от известных параметров (в том числе и от $\Lambda$ ) и не зависят от $s, \delta$. Введем неотрицательные срезающие функции $\eta(\tau) \in C^{m}\left(\mathbb{R}^{1}\right), \zeta(\tau) \in C^{m}\left(\mathbb{R}^{1}\right)$, обладающие следующими свойствами:

$$
\begin{gathered}
\eta(\tau)=1 \quad \forall \tau \leqslant-\frac{15}{16}, \quad \eta(\tau)=0 \quad \forall \tau \geqslant 0, \quad 0 \leqslant \eta(\tau) \leqslant 1 \quad \forall \tau \in \mathbb{R}^{1}, \\
\eta^{\prime}(\tau) \leqslant 0 \quad \forall \tau \in \mathbb{R}^{1}, \quad-\eta^{\prime}(\tau) \geqslant d_{6}>0 \quad \forall \tau \in\left(-\frac{3}{4},-\frac{3}{16}\right), \\
\zeta(\tau)=\frac{1}{16} \quad \forall \tau<\frac{1}{32}, \quad \zeta(\tau)=\tau \quad \forall \tau \geqslant \frac{1}{8}, \quad \zeta^{\prime \prime}(\tau) \geqslant 0 \quad \forall \tau \in \mathbb{R}^{1} .
\end{gathered}
$$

Определим семейство основных срезающих функций следующим образом:

$$
\begin{gathered}
\eta_{s, \delta}(x)=\eta\left((2-i) \zeta\left(\Lambda\left|x^{\prime}\right| \delta^{-1}\right)+\left(\psi_{1}^{i-1} x_{1}+(i-1)\left(\psi^{\prime}, x^{\prime}\right)-s\right) \delta^{-1}\right) \\
\forall s \in \mathbb{R}^{1}, \quad \forall \delta>0, \quad i=1,2 .
\end{gathered}
$$

В силу неравенств (3.2) легко проверяются следующие соотношения:

$$
\begin{gathered}
\eta_{s, \delta}(x)=0 \quad \forall x, \quad \psi_{1}^{i-1} x_{1}+(i-1)\left(\psi^{\prime}, x^{\prime}\right) \geqslant s-(2-i) \delta \zeta\left(\Lambda\left|x^{\prime}\right| \delta^{-1}\right) \\
\eta_{s, \delta}(x)=1 \quad \forall x, \quad \psi_{1}^{i-1} x_{1}+(i-1)\left(\psi^{\prime}, x^{\prime}\right) \leqslant s-\delta\left(\frac{15}{16}+(2-i) \zeta\left(\Lambda\left|x^{\prime}\right| \delta^{-1}\right)\right) \\
\left|D_{x}^{\alpha} \eta_{s, \delta}(x)\right| \leqslant d_{7}|\psi|^{i-1} \delta^{-|\alpha|} \quad \forall \alpha, 1 \leqslant|\alpha| \leqslant m \\
\forall x, \quad s-\delta\left(\frac{15}{16}+(2-i) \zeta\left(\Lambda\left|x^{\prime}\right| \delta^{-1}\right)\right) \leqslant \psi_{1}^{i-1} x_{1}+(i-1)\left(\psi^{\prime}, x^{\prime}\right) \\
\leqslant s-(2-i) \delta \zeta\left(\Lambda\left|x^{\prime}\right| \delta^{-1}\right), \quad d_{7}\left(|\psi|^{i-1}\right)<\infty \\
-D_{x_{1}} \eta_{s, \delta}(x) \geqslant d_{8} \psi_{1}^{i-1} \delta^{-1}>0 \\
\forall x, \quad s-\delta\left(\frac{3}{4}+(2-i) \zeta\left(\Lambda\left|x^{\prime}\right| \delta^{-1}\right)\right) \leqslant \psi_{1}^{i-1} x_{1}+(i-1)\left(\psi^{\prime}, x^{\prime}\right) \\
\leqslant s-\delta\left(\frac{3}{16}+(2-i) \zeta\left(\Lambda\left|x^{\prime}\right| \delta^{-1}\right)\right), \quad d_{8}>0
\end{gathered}
$$


Нетрудно проверить справедливость следующих включений:

$$
\begin{aligned}
& \Omega^{(i)}(s) \supset\left\{x: \psi_{1}^{i-1} x_{1}+(i-1)\left(\psi^{\prime}, x^{\prime}\right) \leqslant s-(2-i) \delta \zeta\left(\Lambda\left|x^{\prime}\right| \delta^{-1}\right)\right\}, \\
& \Omega^{(i)}(s-\delta) \subset\left\{x: \psi_{1}^{i-1} x_{1}+(i-1)\left(\psi^{\prime}, x^{\prime}\right) \leqslant s-\delta\left(\frac{15}{16}+(2-i) \zeta\left(\Lambda\left|x^{\prime}\right| \delta^{-1}\right)\right)\right\}, \\
& K^{(i)}(s, \delta) \equiv \Omega^{(i)}(s) \backslash \Omega^{(i)}(s-\delta) \supset\left\{x: s-\delta\left(\frac{15}{16}+(2-i) \zeta\left(\Lambda\left|x^{\prime}\right| \delta^{-1}\right)\right)\right. \\
&\left.\leqslant \psi_{1}^{i-1} x_{1}+(i-1)\left(\psi^{\prime}, x^{\prime}\right) \leqslant s-(2-i) \delta \zeta\left(\Lambda\left|x^{\prime}\right| \delta^{-1}\right)\right\}, \\
& K^{(i)}\left(s-\frac{\delta}{4}, \frac{\delta}{2}\right) \subset\left\{x: s-\delta\left(\frac{3}{4}+(2-i) \zeta\left(\Lambda\left|x^{\prime}\right| \delta^{-1}\right)\right)\right. \\
&\left.\leqslant \psi_{1}^{i-1} x_{1}+(i-1)\left(\psi^{\prime}, x^{\prime}\right) \leqslant s-\delta\left(\frac{3}{16}+(2-i) \zeta\left(\Lambda\left|x^{\prime}\right| \delta^{-1}\right)\right)\right\},
\end{aligned}
$$

$\forall s \in \mathbb{R}^{1}, \forall \delta>0$. Здесь $\Omega^{(1)}(s) \equiv \Omega^{(1)}(\Lambda, y, s), \Omega^{(2)}(s) \equiv \Omega^{(2)}(y, \psi, s)$, где $\Omega^{(1)}(\Lambda, y, s), \Omega^{(2)}(y, \psi, s)$ - из $(2.1),(2.2)$ соответственно.

Следуя выводу формулы интегрирования по частям из работы [11], из определения 1 выводим для произвольного $T_{0} \leqslant T$ следующее интегральное тождество:

$$
\begin{aligned}
& \frac{q}{q+1} \int_{\Omega}\left|u\left(T_{0}, x\right)\right|^{q+1} \phi\left(T_{0}, x\right) d x+\int_{G_{T_{0}}}\left[-\frac{q}{q+1}|u(t, x)|^{q+1} \phi_{t}^{\prime}(t, x)\right. \\
& \left.\quad+\sum_{|\alpha|=m} a_{\alpha}\left(t, x, u, D_{x} u, \ldots, D_{x}^{m} u\right) D_{x}^{\alpha}(u \cdot \phi)-B(t, u)\left(\chi, \nabla_{x} \phi(t, x)\right)\right] d x d t \\
& =\frac{q}{q+1} \int_{\Omega}\left|u_{0}(x)\right|^{q+1} \phi(0, x) d x, \quad B(t, u)=b(t, u) u-\int_{0}^{u} b(t, s) d s, \quad(3.4)
\end{aligned}
$$

для произвольной функции $\phi(t, x) \in C_{t, x}^{1, m}(\bar{G})$, где $C_{t, x}^{1, m}(\bar{G})$ - множество непрерывно дифференцируемых по $t$ и $m$ раз непрерывно дифференцируемых по $x$ в $\bar{G}$ функций.

Введем следующие обозначения:

$$
\begin{array}{rlrl}
\Psi_{t_{1}}^{t_{2}}(s, h) & =\int_{t_{1}}^{t_{2}}\left(\int_{\Omega(s)}|u|^{q+1} d x\right)^{h} d t & \forall h>0, & E_{T}(s, \tau)=\Psi_{T-\tau}^{T}(s, 1), \\
I_{T}(s, \tau) & =\int_{G_{T-\tau}^{T}(s)}|u|^{p+1} d x d t, & J_{T}(s, \tau)=\int_{G_{T-\tau}^{T}(s)}|u|^{\lambda+1} d x d t, \\
F_{T}(s, \tau)=\int_{G_{T-\tau}^{T}(s)}\left|D_{x}^{m} u\right|^{p+1} d x d t, & H_{T}(s)=\operatorname{ess} \sup _{t \in(0, T)} \int_{\Omega_{t}(s)}|u|^{q+1} d x, \\
I_{T}(s)=I_{T}(s, T), \quad E_{T}(s)=E_{T}(s, T), & J_{T}(s)=J_{T}(s, T), \quad F_{T}(s)=F_{T}(s, T), \\
\Delta I_{T}(s, \delta, \tau)=I_{T}(s, \tau)-I_{T}(s-\delta, \tau), & \Delta J_{T}(s, \delta, \tau)=J_{T}(s, \tau)-J_{T}(s-\delta, \tau), \\
\Delta F_{T}(s, \delta, \tau)=F_{T}(s, \tau)-F_{T}(s-\delta, \tau), & \Delta I_{T}(s, \delta)=I_{T}(s)-I_{T}(s-\delta), \\
\Delta J_{T}(s, \delta)=J_{T}(s)-J_{T}(s-\delta), & \Delta F_{T}(s, \delta)=F_{T}(s)-F_{T}(s-\delta) \\
\forall s \in \mathbb{R}^{1}, & \forall \tau \geqslant 0 .
\end{array}
$$


ЛЕмма 3.1 (см. [27]). Пусть $и(t, x)$ - произвольное энергетическое решение задачи (1.1)-(1.3) при $\chi_{1} \geqslant 0$. Тогда имеет место следующее соотношение:

$$
\begin{aligned}
& H_{T}(s-\delta)+T^{-1} E_{T}(s-\delta)+F_{T}(s-\delta)+\psi_{1} \chi_{1} \delta^{-1} J_{T}\left(s-\frac{5 \delta}{8}, \frac{\delta}{4}\right) \\
& \quad \leqslant c\left(\delta^{-m(p+1)} \Delta I_{T}(s, \delta)+h_{0}(s)\right)
\end{aligned}
$$

$\forall s \in \mathbb{R}^{1}, \forall \delta>0$. Здесъ и далее $c, c_{i}$ - различные положительные постоянные, зависящие лищь от известных параметров задачи.

Лемма 3.2 (см. [27]). Пусть и $(t, x)$ - произвольное энергетическое решение задачи (1.1)-(1.3) при $\chi_{1} \leqslant 0$. Тогда верно следующее соотношение:

$$
\begin{aligned}
& H_{T}(s-\delta)+T^{-1} E_{T}(s-\delta)+F_{T}(s-\delta) \\
& \quad \leqslant c\left(\delta^{-m(p+1)} \Delta I_{T}(s, \delta)-\psi_{1} \chi_{1} \delta^{-1} \Delta J_{T}(s, \delta)+h_{0}(s)\right)
\end{aligned}
$$

$\forall s \in \mathbb{R}^{1}, \forall \delta>0$.

ЛЕмма 3.3. Пусть $и(t, x)$ - произволъное энергетическое решение задачи (1.1)-(1.3) при $\chi_{1}>0$. Тогда выполнено следующее соотношение:

$$
\begin{gathered}
H_{T}(s-\delta)+F_{T}(s-\delta, \tau-\omega)+\psi_{1} \chi_{1} \delta^{-1} \Delta J_{T}(s-\delta, \delta, \tau-\omega) \\
\leqslant c\left(\delta^{-m(p+1)} I_{T}(s, \tau)+\omega^{-1} \Psi_{T-\tau}^{T-\tau+\omega}(s, 1)\right) \equiv R_{T}(s, \delta, \tau, \omega) \\
\forall s \in \mathbb{R}^{1}, \quad \forall \delta>0, \quad \forall 0<\omega<\tau \leqslant T .
\end{gathered}
$$

ДоказАтельство. Подставим в интегральное тождество (3.4) в качестве пробной функции $\varphi(t, x)=\eta_{s, \delta}\left(x_{1}-\delta, x^{\prime}\right) \psi(t)$, где $\psi(t) \in C^{1}\left(\mathbb{R}^{1}\right)$ - срезающая функция такая, что $\psi(t)=0$ при $t \leqslant T-\tau-\omega, \psi(t)=1$ при $t \geqslant T-\tau$, $0 \leqslant \psi(t) \leqslant 1 \forall t>0,|D \psi(t)| \leqslant d_{8} \omega^{-1} \forall 0<\omega<\tau<T, \forall T>\tau+\omega, d_{8}<\infty$. После очевидных преобразований с использованием неравенств (1.4)-(1.7), (3.3), а также свойств функции $\psi(t)$, приходим к следующему неравенству:

$$
\begin{aligned}
& H_{T}(s-\delta)+F_{T}(s-\delta, \tau)+\psi_{1} \chi_{1} \delta^{-1} \Delta J_{T}\left(s+\frac{3 \delta}{4}, \frac{\delta}{2}, \tau\right) \\
& \leqslant c\left(\sum_{|\beta|=0}^{m-1} \delta^{-m+|\beta|} \int_{Q_{T-\tau-\omega}^{T}(s+\delta, \delta)}\left|D_{x}^{m} u\right|^{p}\left|D_{x}^{\beta} u\right| d x d t\right. \\
& \left.\quad+\omega^{-1} \Psi_{T-\tau-\omega}^{T}(s+\delta, 1)\right)
\end{aligned}
$$

$\forall s \in \mathbb{R}^{1}, \forall \delta>0$. Используя для оценки первого слагаемого в правой части (3.8) неравенство Гёльдера, интерполяционное неравенство (3.1) при $a=b=d=$ $p+1$ и неравенство Юнга с $\varepsilon$, получаем соотношение

$$
\begin{gathered}
H_{T}(s)+F_{T}(s, \tau)+\psi_{1} \chi_{1} \delta^{-1} J_{T}\left(s+\frac{3 \delta}{4}, \frac{\delta}{2}, \tau\right) \leqslant \varepsilon \Delta F_{T}(s+\delta, \delta, \tau+\omega) \\
+c(\varepsilon)\left(\delta^{-m(p+1)} \Delta I_{T}(s+\delta, \delta, \tau+\omega)+\omega^{-1} E_{T}(s+\delta, \tau+\omega)\right) \\
\forall s \in \mathbb{R}^{1}, \quad \forall \delta>0, \quad \forall \varepsilon>0 .
\end{gathered}
$$

Зафиксируем произвольно $s=s_{0}, \delta=\delta_{0}, \tau=\tau_{0}, \omega=\omega_{0}$ и определим последовательности $s_{i}=s_{i-1}+\delta_{i-1}, \delta_{i}=2^{-i} \delta_{0}, \quad \tau_{i}=\tau_{i-1}+\omega_{i-1}, \quad \omega_{i}=2^{-i} \omega_{0}$, 
$i=1,2, \ldots$ Из (3.9) получим теперь серию соотношений, фиксируя каждый раз $s=s_{i}, \delta=\delta_{i}, \tau=\tau_{i}, \omega=\omega_{i}$. Итерируя эти соотношения $k$ раз, получаем

$$
\begin{aligned}
H_{T}(s) & +F_{T}(s, \tau)+\psi_{1} \chi_{1} \delta^{-1} \Delta J_{T}\left(s+\frac{3 \delta}{4}, \frac{\delta}{2}, \tau\right) \leqslant \varepsilon^{k+1} \Delta F_{T}(s+2 \delta, 2 \delta, \tau+2 \omega) \\
& +c(\varepsilon)\left(\omega^{-1} \sum_{j=0}^{k}(2 \varepsilon)^{j} \Psi_{T-\tau-2 \omega}^{T}(s+2 \delta, 1)\right. \\
& \left.+\sum_{j=0}^{k}\left(2^{m(p+1)} \varepsilon\right)^{j} \delta^{-m(p+1)} \Delta I_{T}(s+2 \delta, 2 \delta, \tau+2 \omega)\right)
\end{aligned}
$$

$\forall s \in \mathbb{R}^{1}, \forall \delta>0$. Полагая здесь $\varepsilon=2^{-m(p+1)-1}$, устремляя $k \rightarrow \infty$ и заменяя в полученном неравенстве аргументы, приходим к неравенству

$$
\begin{aligned}
& H_{T}(s-\delta)+F_{T}(s-\delta, \tau-\omega)+\psi_{1} \chi_{1} \delta^{-1} \Delta J_{T}\left(s-\frac{5 \delta}{8}, \frac{\delta}{4}, \tau-\omega\right) \\
& \quad \leqslant c\left(\delta^{-m(p+1)} \Delta I_{T}(s, \delta, \tau)+\omega^{-1} \Psi_{T-\tau}^{T-\tau+\omega}(s, 1)\right) \quad \forall s \in \mathbb{R}^{1}, \quad \forall \delta>0 .
\end{aligned}
$$

Записывая это неравенство для $s=s_{0}, s_{i}=s_{i-1}-\delta / 4, i=1,2, \ldots, 6$, и складывая полученные соотношения, приходим к требуемому соотношению. Лемма доказана.

Лемма 3.4. Пусть $и(t, x)$ - произвольное энергетическое решение задачи (1.1)-(1.3) при $\chi_{1}>0$. Тогда имеет место следующее соотношение:

$$
\Psi_{T-\tau+\omega}^{T}\left(s-\delta, h_{2}\right) \leqslant c \Psi_{T-\tau}^{T}\left(s, h_{1}\right) R_{T}(s, \delta, \tau, \omega)^{h_{2}-h_{1}}
$$

$\forall 0<h_{1}<h_{2}<\infty, \forall s \in \mathbb{R}^{1}, \forall \delta>0, \forall \tau, \omega, 0<\omega<\tau<T$.

ДокАЗАТЕЛьСТво. Введем вспомогательную функцию

$$
X_{h}(t)=\int_{0}^{t}\left(\int_{\Omega(s)}|u(x, \theta)|^{q+1} \eta_{s, \delta}(x) \psi(\theta-\omega) d x\right)^{h} d \theta
$$

где $\psi(t)$ - функция из леммы 3.3. В силу теоремы Фубини имеет место соотношение

$$
\Phi_{T-\tau}^{T}(s, h+1) \equiv X_{h+1}(T)=\int_{G_{T-\tau}^{T}(s)}|u|^{q+1} \eta_{s, \delta}(x) \psi(t-\omega) \frac{d}{d t} X_{h}(t) d x d t .
$$

Подставляя в интегральное тождество (3.4) в качестве срезающей функции

$$
\varphi(x, t)=\psi(t-\omega) X_{h}(t) \eta_{s, \delta}(x),
$$

получаем, учитывая неравенство (3.12), следующее соотношение:

$$
\begin{aligned}
& \Phi_{T-\tau}^{T}(s, h+1)=\Phi_{T-\tau}^{T}(s, h) \int_{\Omega_{T}(s)}|u|^{q+1} \eta_{s, \delta}(x) d x \\
& \quad+\frac{q+1}{q} \int_{G_{T-\tau}^{T}(s)}\left[\sum_{|\alpha|=m} a_{\alpha}\left(t, x, u, D_{x} u, \ldots, D_{x}^{m} u\right) D_{x}^{\alpha}\left(u \eta_{s, \delta}(x)\right)\right.
\end{aligned}
$$




$$
\begin{aligned}
& \left.-\chi_{1} B(t, u) D_{x_{1}} \eta_{s, \delta}(x)\right] \psi(t-\omega) X_{h}(t) d x d t \\
& -\int_{G_{T-\tau}^{T-\tau+\omega}(s)}|u|^{q+1} \eta_{s, \delta} \psi^{\prime}(t-\omega) X_{h}(t) d x d t .
\end{aligned}
$$

После преобразований с использованием оценок (1.5)-(1.7), свойств функций $\psi(t), X_{h}(t)$, неравенства Гёльдера, интерполяционного неравенства (3.1) при $a=b=d=p+1$, неравенства Юнга и соотношения (3.7), приходим к следующему выражению

$$
\Phi_{T-\tau}^{T}(s, h+1) \leqslant c \Phi_{T-\tau}^{T}(s, h) R_{T}(s+\delta, \delta, \tau+\omega, \omega) \quad \forall h>0 .
$$

Итерируя последнее неравенство, получаем, что

$$
\Phi_{T-\tau}^{T}(s, h) \leqslant c \Phi_{T-\tau}^{T}(s, h-l) R_{T}(s+\delta, \delta, \tau+\omega, \omega)^{l} \quad \forall h>0, \quad \forall h>l \in \mathbb{N},
$$

откуда в силу неравенства Гёльдера вытекает соотношение

$$
\Phi_{T-\tau}^{T}\left(s, h_{2}\right) \leqslant c \Phi_{T-\tau}^{T}\left(s, h_{1}\right) R_{T}(s+\delta, \delta, \tau+\omega, \omega)^{h_{2}-h_{1}} \quad \forall 0<h_{1}<h_{2} .
$$

Заметим, что справедливо следующее очевидное неравенство:

$$
\Psi_{T-\tau+\omega}^{T}(s-\delta, h) \leqslant \Phi_{T-\tau}^{T}(s, h) \leqslant \Psi_{T-\tau}^{T}(s, h) .
$$

Отсюда с учетом неравенства (3.13) получаем, что

$$
\Psi_{T-\tau+\omega}^{T}\left(s-\delta, h_{2}\right) \leqslant c \Psi_{T-\tau}^{T}\left(s+\delta, h_{1}\right) R_{T}(s+\delta, \delta, \tau+\omega, \omega)^{h_{2}-h_{1}} .
$$

После замены аргументов в последнем соотношении приходим к (3.11). Лемма доказана.

\section{§ 4. Прямое движение левого фронта}

ДоКАЗАТЕЛЬСТво ТЕОРЕмЫ 1. Из интерполяционного неравенства (3.1) при $\delta \rightarrow \infty, j=0, a=b=p+1, d=q+1$ следует, что

$$
\int_{\Omega(s)}|u|^{p+1} d x \leqslant d_{5}^{p+1}\left(\int_{\Omega(s)}\left|D_{x}^{m} u\right|^{p+1} d x\right)^{\theta}\left(\int_{\Omega(s)}|u|^{q+1} d x\right)^{\frac{p+1}{q+1}(1-\theta)} \forall s \in \mathbb{R}^{1},
$$

где $\theta=\frac{n(p-q)}{m(p+1)(q+1)+n(p-q)}$. Интегрируя (4.1) по $t$ и применяя неравенство Гёльдера, приходим к соотношению

$$
I_{T}(s) \leqslant c F_{T}(s)^{\theta} \Psi_{0}^{T}\left(s, \frac{p+1}{q+1}\right)^{1-\theta} \quad \forall s \in \mathbb{R}^{1},
$$

откуда следует неравенство

$$
I_{T}(s) \leqslant c F_{T}(s)^{\theta} E_{T}(s)^{1-\theta} H_{T}(s)^{\frac{p-q}{q+1}(1-\theta)}
$$

$\forall s \in \mathbb{R}^{1}$. Из (4.3) в силу леммы 3.1 вытекает неравенство

$$
\begin{gathered}
I_{T}(s-\delta) \leqslant c_{1} T^{1-\theta}\left(\delta^{-m(p+1)} \Delta I_{T}(s)+h(s)\right)^{1+\alpha} \quad \forall s \in \mathbb{R}^{1}, \quad \forall \delta>0, \\
\alpha=\frac{m(p+1)(p-q)}{n(p-q)+m(p+1)(q+1)} .
\end{gathered}
$$


Полагая в неравенстве $(4.4) \delta=\delta(s, T)=T^{\frac{1-\theta}{m(p+1)(\alpha+1)}} I_{T}(s)^{\frac{\alpha}{m(p+1)(\alpha+1)}}$, после очевидных преобразований приходим к соотношению

$$
I_{T}(s-\delta(s, T)) \leqslant c_{2}\left(I_{T}(s)+T^{1-\theta} h_{0}(s)^{1+\alpha}\right) \quad \forall s \in \mathbb{R}^{1}, \quad c_{2}=c_{1}\left(c_{1}+1\right)^{-1}<1 .
$$

Возведя обе части последнего неравенства в степень $\alpha /(m(p+1)(1+\alpha))$ и умножив затем на $T^{\frac{1-\theta}{m(p+1)(1+\alpha)}}$, получим следующее соотношение:

$$
P_{T}\left(s-P_{T}(s)\right) \leqslant \kappa_{1}\left(P_{T}(s)+T^{\frac{1-\theta}{m(p+1)}} h_{0}(s)^{\frac{\alpha}{m(p+1)}}\right) \quad \forall s \in \mathbb{R}^{1},
$$

$P_{T}(s) \equiv \delta(s, T), \kappa_{1}=c_{2}^{\frac{\alpha}{m(p+1)(1+\alpha)}}<1$.

Вернемся теперь к соотношению (4.4). Полагая в нем $\delta=2^{-1} s$ и устремляя в полученном неравенстве $s \rightarrow \infty$, в силу ограниченности и монотонности функций $I_{T}(s), h_{0}(s)$ для функции $P_{T}(s)$ получаем следующее соотношение:

$$
P_{T}(\infty) \leqslant c_{3} T^{\frac{1-\theta}{m(p+1)}} h_{0}(\infty)^{\frac{\alpha}{m(p+1)}}, \quad c_{3}=c_{1}^{\frac{\alpha}{m(p+1)(1+\alpha)}} .
$$

Из (4.6) следует, что существует $s_{1}<\infty$ такое, что выполнено

$$
P_{T}(s) \leqslant L \kappa_{1} T^{\frac{1-\theta}{m(p+1)}} h_{0}(s)^{\frac{\alpha}{m(p+1)}} \quad \forall s>s_{1}, \quad L>\max \left\{c_{3} \kappa_{1}^{-1},\left(1-\kappa_{1}\right)^{-1}\right\} .
$$

Обратимся к неравенству (4.5). Из него, очевидно, следует соотношение

$$
P_{T}\left(s-P_{T}(s)\right) \leqslant \kappa_{1} P_{T}(s) \quad \forall s \leqslant 0 .
$$

Из (4.8) в силу леммы 8.1 из дополнения имеем

$$
P_{T}(s) \equiv 0 \quad \forall s \leqslant s_{0}-\left(1-\kappa_{1}\right)^{-1} P_{T}\left(s_{0}\right), \quad \forall s_{0} \leqslant 0
$$

или, иначе,

$$
\eta_{-}(T) \geqslant s_{0}-\left(1-\kappa_{1}\right)^{-1} T^{\frac{1-\theta}{m(p+1)(1+\alpha)}} I_{T}\left(s_{0}\right)^{\frac{\alpha}{m(p+1)(1+\alpha)}} \quad \forall s_{0} \leqslant 0 .
$$

Следствием неравенства (4.9) является следующее соотношение:

$$
\eta_{-}(T) \geqslant-\left(1-\kappa_{1}\right)^{-1} P_{T}(0) .
$$

Из неравенств (4.5), (4.7) в силу леммы 8.2 из дополнения получаем, что

$$
P_{T}(0) \leqslant c T^{\frac{1-\theta}{m(p+1)}} h\left(\widetilde{v}_{1}(T)\right)^{\frac{\alpha}{m(p+1)}},
$$

где $\widetilde{v}_{1}(T)=\inf \left\{T^{\frac{1-\theta}{m(p+1)}} h(s)^{\frac{\alpha}{m(p+1)}}<\kappa_{2} s\right\}, \kappa_{2}=\left(L \kappa_{1}\right)^{-1}\left(1-\kappa_{1}-L^{-1}\right)$. Оценивая правую часть (4.11) с помощью (4.12), получаем, что

$$
\eta_{-}(T) \geqslant-c T^{\beta_{1}} h\left(\widetilde{v}_{1}(T)\right)^{\frac{p-q}{q+1} \beta_{1}} \quad \forall T>0 .
$$

Оценим теперь $I_{T}(s)$ иначе. Заметим предварительно, что при

$$
\Omega(s)=\Omega(\Lambda, y, s)
$$

справедливо следующее неравенство:

$$
\operatorname{mes}\{\Omega(s) \cap \operatorname{supp} u(\cdot, t)\} \leqslant c(\Lambda)\left(s-\eta_{-}(t)\right)_{+}^{n}, \quad c(\Lambda)<\infty, \quad a_{+}=\max \{a, 0\} .
$$


Полагая в $(3.5) s=s_{0}+\delta, s_{i}=s_{i-1}-\delta / 4, i=1,2 \ldots$, и суммируя полученные неравенства, приходим к соотношению

$$
F_{T}(s)+\chi_{1} \delta^{-1} J_{T}(s) \leqslant C \delta^{-m(p+1)} I_{T}(s+\delta) \quad \forall \delta>0, \quad s+\delta \leqslant 0 .
$$

Применяя к правой части (4.15) неравенство Юнга с $\varepsilon$ и оценивая правую часть полученного выражения с помощью (4.14), приходим к соотношению

$$
F_{T}(s)+\chi_{1} \delta^{-1} J_{T}(s) \leqslant \varepsilon \chi_{1} \delta^{-1} J_{T}(s+\delta)+\chi_{1}^{-\frac{p+1}{\lambda-p}} c(\varepsilon) T\left(-\eta_{-}(T)\right)^{n} \delta^{-m(p+1) \frac{\lambda+1}{\lambda-p}+\frac{p+1}{\lambda-p}}
$$

$\forall \delta>0, s+\delta \leqslant 0, \forall \varepsilon>0$. Применяя к неравенству (4.16) итеративную процедуру, аналогичную представленной в доказательстве леммы 3.3 , приходим к следующему соотношению:

$$
F_{T}(s) \leqslant c T\left(-\eta_{-}(T)\right)^{n}(-s)^{-m(p+1) \frac{\lambda+1}{\lambda-p}+\frac{p+1}{\lambda-p}} \chi_{1}^{-\frac{p+1}{\lambda-p}} \quad \forall s<0 .
$$

В силу неравенства Пуанкаре

$$
I_{T}\left(s_{0}\right) \leqslant c\left(-\eta_{-}(T)\right)^{m(p+1)} F_{T}\left(s_{0}\right) \quad \forall s_{0} \leqslant 0 .
$$

Оценивая правую часть (4.18) с помощью (4.17), получаем, что

$$
I_{T}\left(s_{0}\right) \leqslant c \chi_{1}^{-\frac{p+1}{\lambda-p}} T\left(-\eta_{-}(T)\right)^{n+m(p+1)}\left(-s_{0}\right)^{-\frac{(m(\lambda+1)-1)(p+1)}{\lambda-p}} \quad \forall s_{0}<0 .
$$

Из (4.10) в силу (4.19) вытекает, что

$$
\begin{aligned}
\eta_{-}(T) \geqslant s_{0} & -c \chi_{1}^{-\frac{\alpha(p+1)}{m(p+1)(\alpha+1)(\lambda-p)}} T^{\frac{1-\theta+\alpha}{m(p+1)(\alpha+1)}}\left(-\eta_{-}(T)\right)^{\frac{\alpha(n+m(p+1))}{m(p+1)(\alpha+1)}} \\
& \times\left(-s_{0}\right)^{-\frac{\alpha(m(\lambda+1)-1)}{m(\lambda-p)(1+\alpha)}} \equiv \varphi\left(s_{0}\right) .
\end{aligned}
$$

Оптимизируя функцию $\varphi(s)$, получаем соотношение

$$
\begin{gathered}
\max _{-\infty<s_{0}<0} \varphi\left(s_{0}\right)=\varphi\left(s_{\mathrm{opt}}\right), \quad s_{\mathrm{opt}}=-c T^{\gamma_{1}}\left(-\eta_{-}(T)\right)^{\gamma_{2}} \chi_{1}^{-\gamma_{3}}, \\
\gamma_{1}=\frac{(1-\theta+\alpha)(\lambda-p)}{(p+1)((m(\lambda+1)-1) \alpha+m(\lambda-p)(\alpha+1))}, \\
\gamma_{2}=\frac{\alpha(\lambda-p)(n+m(p+1))}{(p+1)((m(\lambda+1)-1) \alpha+m(\lambda-p)(\alpha+1))}, \\
\gamma_{3}=\frac{\alpha}{(p+1)((m(\lambda+1)-1) \alpha+m(\lambda-p)(\alpha+1))} .
\end{gathered}
$$

Отсюда, очевидно, следует неравенство

$$
\eta_{-}(T) \geqslant-c T^{\frac{\gamma_{1}}{1-\gamma_{2}}} \chi_{1}^{-\frac{\gamma_{3}}{1-\gamma_{2}}}=-c T^{\beta_{2}} \chi_{1}^{-\frac{p-q}{\lambda-p} \beta_{2}} \quad \forall T>0 .
$$

Утверждение теоремы 1 вытекает из оценок (4.13) и (4.20) в силу правильной монотонности функции $h(s)$. Теорема 1 доказана.

ДокАЗАТЕЛЬСтво ТЕОРЕмЫ 2. Справедливость оценки (4.13) при $q<p$ доказана в теореме 1. Выведем теперь оценку (2.21). Из (3.5), очевидно, вытекает соотношение

$$
\Delta I_{T}\left(s-\frac{5 \delta}{4}, \frac{\delta}{4}\right) \leqslant c\left(\psi_{1} \chi_{1}\right)^{-1} \delta^{1-m(p+1)} \Delta I_{T}\left(s, \frac{\delta}{4}\right) \quad \forall s \leqslant 0, \quad \forall \delta>0 .
$$


Полагая в (4.21) последовательно $s_{i}=s-i \delta / 4, i=0,1,2, \ldots$, и складывая полученные неравенства, приходим к соотношению

$$
I_{T}(s-\delta) \leqslant c\left(\psi_{1} \chi_{1}\right)^{-1} \delta^{1-m(p+1)} I_{T}(s) \quad \forall s \leqslant 0, \quad \forall \delta>0 .
$$

Зафиксируем теперь $\delta$ следующим образом:

$$
\begin{gathered}
c\left(\psi_{1} \chi_{1}\right)^{-1} \delta^{1-m(p+1)}=2^{-1} \Rightarrow \delta=\left(2 c\left(\psi_{1} \chi_{1}\right)^{-1}\right)^{\gamma_{7}}=h=\mathrm{const}, \\
\gamma_{7}=(m(p+1)-1)^{-1},
\end{gathered}
$$

откуда, очевидно, следует, что $I_{T}(s-h) \leqslant 2^{-1} I_{T}(s) \forall s \leqslant 0$. Итерируя предыдущее неравенство $j$ раз, получаем, что

$$
I_{T}(s-j h) \leqslant 2^{-j} I_{T}(s) \quad \forall s \leqslant 0, \quad \forall j \in \mathbb{N},
$$

откуда приходим к соотношению

$$
I_{T}\left(s_{2}\right) \leqslant 2 \exp \left(-c\left(\psi_{1} \chi_{1}\right)^{\gamma_{7}}\left(s_{1}-s_{2}\right)\right) I_{T}\left(s_{1}\right) \quad \forall s_{2}<s_{1} \leqslant 0
$$

или, иначе,

$$
I_{T}\left(s_{0}\right) \leqslant 2 \exp \left(c\left(\psi_{1} \chi_{1}\right)^{\gamma_{7}} s_{0}\right) I_{T}(0) \quad \forall s_{0} \leqslant 0 .
$$

Из (4.10) в силу предыдущего неравенства вытекает, что

$$
\eta_{-}(T) \geqslant s_{0}-c T^{\frac{1-\theta}{m(p+1)(1+\alpha)}} I_{T}(0)^{\frac{\alpha}{m(p+1)(1+\alpha)}} \exp \left(c\left(\psi_{1} \chi_{1}\right)^{\gamma_{7}} s_{0}\right) \quad \forall s_{0} \leqslant 0 .
$$

Полагая в $(3.5) \delta=2^{-1} s$ и устремляя в полученном соотношении $\delta \rightarrow \infty$, приходим к следующему неравенству:

$$
F_{T}(s) \leqslant c h_{0}(\infty) \quad \forall s \leqslant 0 .
$$

Из (4.18) в силу последнего соотношения следует, что

$$
I_{T}(0) \leqslant C\left(-\eta_{-}(T)\right)^{m(p+1)} .
$$

Оценим (4.22) с помощью (4.23). Имеем

$$
\eta_{-}(T) \geqslant s_{0}-C T^{\frac{1-\theta}{m(p+1)(1+\alpha)}} \exp \left(c\left(\psi_{1} \chi_{1}\right)^{\gamma_{7}} s_{0}\right)\left(-\eta_{-}(T)\right)^{\frac{\alpha}{1+\alpha}} \quad \forall s_{0} \leqslant 0,
$$

откуда в силу неравенства Юнга с $\varepsilon$, очевидно, вытекает, что

$$
\eta_{-}(T) \geqslant \varepsilon\left(\eta_{-}(T)\right)+s_{0}-c T^{\frac{1-\theta}{m(p+1)}} \exp \left(c\left(\psi_{1} \chi_{1}\right)^{\gamma_{7}(1+\alpha)} s_{0}\right) \quad \forall s_{0} \leqslant 0 .
$$

Полагая в предыдущем неравенстве $\varepsilon=2^{-1}$, приходим к соотношению

$$
\eta_{-}(T) \geqslant 2\left(s_{0}-C T^{\frac{1-\theta}{m(p+1)}} \exp \left(c\left(\psi_{1} \chi_{1}\right)^{\gamma_{7}(1+\alpha)} s_{0}\right)\right) \equiv \varphi\left(s_{0}\right) .
$$

Оптимизировав функцию $\varphi(s)$, найдем $s_{\text {opt }}: \varphi\left(s_{\text {opt }}\right)=\max _{s_{0}<0} \varphi\left(s_{0}\right)$. Имеем

$$
s_{\mathrm{opt}}=c\left(\psi_{1} \chi\right)_{1}^{-\gamma_{7}(1+\alpha)}\left(-C-\frac{1-\theta}{m(p+1)} \ln \left(\left(\psi_{1} \chi_{1}\right)^{-\gamma_{7}(1+\alpha)} T\right)\right)<0,
$$

откуда

$$
\eta_{-}(T) \geqslant-C\left(\psi_{1} \chi_{1}\right)^{-\gamma_{7}(1+\alpha)} \ln \left(\left(\psi_{1} \chi_{1}\right)^{-\gamma_{7}(1+\alpha)} T\right) \quad \forall T \geqslant T^{*}\left(\chi_{1}\right)>0 .
$$

Теорема доказана. 
ДокАЗАТЕЛЬСтво теоремы 3. Докажем сначала наличие ЗРН-эффекта при выполнении условия малости (2.25). Пусть в (1.1) выполнено условие $q<\lambda<p$. Из интерполяционного неравенства (3.1) при $a=b=p+1, d=\lambda+1$, $\delta \rightarrow \infty$ вытекает, что

$$
\begin{gathered}
\int_{\Omega(s)}|u|^{p+1} d x \leqslant d_{5}^{p+1}\left(\int_{\Omega(s)}\left|D_{x}^{m} u\right|^{p+1} d x\right)^{\theta_{1}}\left(\int_{\Omega(s)}|u|^{\lambda+1} d x\right)^{\left(1-\theta_{1}\right) \frac{p+1}{\lambda+1}}, \\
\theta_{1}=\frac{n(p-\lambda)}{n(p-\lambda)+m(p+1)(\lambda+1)} .
\end{gathered}
$$

Из того же неравенства при $\delta \rightarrow \infty, a=\lambda+1, b=p+1, d=q+1$ следует, что

$$
\begin{gathered}
\int_{\Omega(s)}|u|^{\lambda+1} d x \leqslant d_{5}^{\lambda+1}\left(\int_{\Omega(s)}\left|D_{x}^{m} u\right|^{p+1} d x\right)^{\theta_{2} \frac{\lambda+1}{p+1}}\left(\int_{\Omega(s)}|u|^{q+1} d x\right)^{\left(1-\theta_{2}\right) \frac{\lambda+1}{q+1}}, \\
\theta_{2}=\frac{n(p+1)(\lambda-q)}{(\lambda+1)(n(p-q)+m(p+1)(q+1))} .
\end{gathered}
$$

Оценивая правую часть (4.24) с помощью (4.25), приходим к следующему неравенству:

$$
\begin{gathered}
\int_{\Omega(s)}|u|^{p+1} d x \leqslant c\left(\int_{\Omega(s)}\left|D_{x}^{m} u\right|^{p+1} d x\right)^{\theta_{1}+h \theta_{2} \frac{\lambda+1}{p+1}} \\
\times\left(\int_{\Omega(s)}|u|^{\lambda+1} d x\right)^{\frac{p+1}{\lambda+1}\left(1-\theta_{1}\right)-h}\left(\int_{\Omega(s)}|u|^{q+1} d x\right)^{\frac{\lambda+1}{q+1}\left(1-\theta_{2}\right) h} \\
\forall s \in \mathbb{R}^{1}, \quad 0 \leqslant h<(p+1)(\lambda+1)^{-1}\left(1-\theta_{1}\right) .
\end{gathered}
$$

Зафиксируем теперь $h$ так, чтобы было выполнено соотношение

$$
\theta_{1}+h \theta_{2}(\lambda+1)(p+1)^{-1}+\left(1-\theta_{1}\right)(p+1)(\lambda+1)^{-1}-h=1 .
$$

Оно справедливо при

$$
h=\frac{m(p+1)(p-\lambda)}{m(p+1)(\lambda+1)+n(p-\lambda)} \cdot \frac{n(p-q)+m(p+1)(q+1)}{n(p-\lambda)+m(p+1)(q+1)} .
$$

Подставив это значение $h$ в (4.26), получим, что

$$
\int_{\Omega(s)}|u|^{p+1} d x \leqslant c\left(\int_{\Omega(s)}\left|D_{x}^{m} u\right|^{p+1} d x\right)^{\nu}\left(\int_{\Omega(s)}|u|^{\lambda+1} d x\right)^{1-\nu}\left(\int_{\Omega(s)}|u|^{q+1} d x\right)^{\mu}
$$

$\forall s \in \mathbb{R}^{1}$, где $\nu, \mu-$ из (2.23). Интегрируя (4.27) по $t$ и применяя неравенство Гёльдера, приходим к следующему соотношению:

$$
I_{T}(s) \leqslant c F_{T}(s)^{\nu} J_{T}(s)^{1-\nu} H_{T}(s)^{\mu} \quad \forall s \in \mathbb{R}^{1} .
$$

Выведем теперь соотношение (4.28) в случае $\lambda<q<p$. В силу интерполяционного неравенства (3.1) при $\delta \rightarrow \infty, a=q+1, b=p+1, d=\lambda+1$ имеем

$$
\begin{gathered}
\int_{\Omega(s)}|u|^{q+1} d x \leqslant d_{5}^{q+1}\left(\int_{\Omega(s)}\left|D_{x}^{m} u\right|^{p+1} d x\right)^{\theta_{3} \frac{q+1}{p+1}}\left(\int_{\Omega(s)}|u|^{\lambda+1} d x\right)^{\left(1-\theta_{3}\right) \frac{q+1}{\lambda+1}} \\
\theta_{3}=\frac{n(p+1)(q-\lambda)}{(q+1)(n(p-\lambda)+m(p+1)(\lambda+1))}<1 .
\end{gathered}
$$


Возводя обе части неравенства (4.29) в степень

$$
\xi=\left(\frac{\theta_{3}(q+1)}{p+1}+\frac{\left(1-\theta_{3}\right)(q+1)}{\lambda+1}\right)^{-1}=\frac{n(p-\lambda)+m(p+1)(\lambda+1)}{n(p-\lambda)+m(p+1)(q+1)}<1,
$$

интегрируя по $t$ и применяя неравенство Гёльдера, получаем соотношение

$$
\Psi_{0}^{T}(s, \xi) \leqslant c F_{T}(s)^{\nu_{1}} J_{T}(s)^{1-\nu_{1}} \quad \forall s \in \mathbb{R}^{1},
$$

где $\nu_{1}-$ из (2.31). Из (4.2), очевидно, следует неравенство

$$
I_{T}(s) \leqslant F_{T}(s)^{\theta} \Psi_{T}(s, \xi)^{1-\theta} H_{T}(s)^{(1-\theta)\left(\frac{p+1}{q+1}-\xi\right)} \quad \forall s \in \mathbb{R}^{1} .
$$

Применяя теперь для оценки второго сомножителя в правой части предыдущего выражения неравенство (4.30), приходим к неравенству (4.28).

В случае $\lambda=q<p$ неравенство (4.28) эквивалентно неравенству (4.3).

Обратимся теперь к соотношению (3.5). Полагая в нем $s=s_{0}, s_{i}=s_{i-1}-$ $4^{-1} \delta, i=1,2, \ldots$, и суммируя полученные соотношения, приходим к неравенству

$$
J_{T}(s-\delta) \leqslant c\left(\psi_{1} \chi_{1}\right)^{-1} \delta^{1-m(p+1)}\left(I_{T}(s)+\delta \sum_{i=0}^{\infty} h_{0}\left(s-i \frac{\delta}{4}\right)\right) .
$$

В силу монотонности $h_{0}(s)$ справедлива следующая цепочка неравенств:

$$
\delta \sum_{i=0}^{\infty} h_{0}\left(s-i \frac{\delta}{4}\right) \leqslant 4 \int_{-\infty}^{s+4^{-1} \delta} h_{0}(\theta) d \theta \leqslant 4\left(s+\frac{\delta}{4}\right) h_{0}\left(s+\frac{\delta}{4}\right) .
$$

Оценивая с помощью последнего соотношения правую часть (4.31) и производя в полученном неравенстве замену аргументов, приходим к соотношению

$$
J_{T}(s-\delta) \leqslant c\left(\psi_{1} \chi_{1}\right)^{-1}\left(\delta^{1-m(p+1)} I_{T}(s)+s h_{0}(s)\right) .
$$

Применяя для оценки правой части (4.28) неравенства (3.5) и (4.32), получаем, что

$$
I_{T}(s-\delta) \leqslant c\left(\psi_{1} \chi_{1}\right)^{\nu-1}\left(\delta^{-m(p+1)} I_{T}(s)+h_{0}(s)\right)^{\nu+\mu}\left(\delta^{1-m(p+1)} I_{T}(s)+s h_{0}(s)\right)^{1-\nu},
$$

откуда, очевидно, следует неравенство

$$
\begin{aligned}
I_{T}(s-\delta) \leqslant c & \left(\psi_{1} \chi_{1}\right)^{\nu-1}\left(\delta^{-m(p+1)(1+\mu)+1-\nu} I_{T}(s)^{1+\mu}\right. \\
+ & \delta^{-m(p+1)(\nu+\mu)} I_{T}(s)^{\nu+\mu} h_{0}(s)^{1-\nu} s^{1-\nu} \\
+ & \left.\delta^{(1-m(p+1))(1-\nu)} I_{T}(s)^{1-\nu} h_{0}(s)^{\nu+\mu}+s^{1-\nu} h_{0}(s)^{1+\mu}\right) \\
& \quad \forall s \in \mathbb{R}^{1}, \quad \forall \delta>0 .
\end{aligned}
$$

Оценив правую часть последнего выражения с помощью неравенства Юнга, получим, что

$$
\begin{gathered}
I_{T}(s-\delta) \leqslant c\left(\psi_{1} \chi_{1}\right)^{\nu-1}\left(\delta^{-m(p+1)(1+\mu)+1-\nu} I_{T}(s)^{1+\mu}\right. \\
\left.+\delta^{-m(p+1)(1+\mu)} s_{+}^{1-\nu} I_{T}(s)^{1+\mu}+s^{1-\nu} h_{0}(s)^{1+\mu}\right) \\
\forall s \in \mathbb{R}^{1}, \quad \forall \delta>0 .
\end{gathered}
$$


Положим в последнем неравенстве

$$
\begin{aligned}
\delta=\delta & \left(s, T, \chi_{1} \psi_{1}\right) \equiv\left(\left(4 c\left(\psi_{1} \chi_{1}\right)^{\nu-1}\right)^{\mu^{-1}} I_{T}(s)\right)^{\frac{\mu}{m(p+1)(1+\mu)+\nu-1}} \\
& +\left(\left(4 c\left(\psi_{1} \chi_{1}\right)^{\nu-1} s^{1-\nu}\right)^{\mu^{-1}} I_{T}(s)\right)^{\frac{\mu}{m(p+1)(1+\mu)}} \quad \forall s>0 .
\end{aligned}
$$

В результате имеем

$$
I_{T}\left(s-\delta\left(s, T, \psi_{1} \chi_{1}\right)\right) \leqslant 2^{-1} I_{T}(s)+c\left(\psi_{1} \chi_{1}\right)^{\nu-1} s^{1-\nu} h_{0}(s)^{1+\mu} \quad \forall s \in \mathbb{R}^{1} .
$$

Возведя обе части (4.34) в степень $\mu \beta_{3}, \beta_{3}-$ из $(2.23)$, и умножив затем на $\left(4 c\left(\psi_{1} \chi_{1}\right)^{\nu-1}\right)^{\beta_{3}}$, для функции $R_{T}(s)=\left(\left(4 c\left(\psi_{1} \chi_{1}\right)^{\nu-1}\right)^{\mu^{-1}} I_{T}(s)\right)^{\mu \beta_{3}}$ получим следующее соотношение:

$$
R_{T}\left(s-R_{T}(s)-R_{T}(s)^{1-\beta_{4}} s^{\beta_{4}}\right) \leqslant \kappa_{3} R_{T}(s)+f(s) \quad \forall s>0,
$$

$\beta_{4}$ - из $(2.23), \kappa_{3}=2^{-\mu \beta_{3}}<1, f(s)=c_{4}\left(\psi_{1} \chi_{1}\right)^{-(1+\mu)(1-\nu) \beta_{3}}\left(s^{1-\nu} h_{0}(s)^{1+\mu}\right)^{\mu \beta_{3}}$. Из априорной оценки (4.6) следует, что

$$
\begin{aligned}
I_{T}(s) & \leqslant c T^{1-\theta} h_{0}(\infty)^{1+\alpha} \quad \forall s \in \mathbb{R}^{1} \\
& \Rightarrow \quad R_{T}(s) \leqslant C T^{\mu(1-\theta) \beta_{3}}\left(\psi_{1} \chi_{1}\right)^{(\nu-1) \beta_{3}} \quad \forall s \in \mathbb{R}^{1} .
\end{aligned}
$$

$\mathrm{K}$ функции $R_{T}(s)$ применим лемму 8.2 из дополнения (§ 8). Проверим сначала условие (8.7). Очевидно, что

$$
R_{T}\left(s_{0}\right) \leqslant C T^{\mu(1-\theta) \beta_{5}}\left(\psi_{1} \chi_{1}\right)^{(\nu-1) \beta_{5}} \leqslant 2\left(1-\kappa_{3}\right)^{-1} f\left(s_{0}\right),
$$

если выполнено

$$
T=T\left(s_{0}\right)<C\left(\left(\psi_{1} \chi_{1}\right)^{-1} s_{0}^{1-\nu} h_{0}\left(s_{0}\right)^{1+\mu}\right)^{(1-\theta)^{-1}} .
$$

Из соотношения (4.35) при выполнении условия (4.36) в силу леммы 8.2 следует, чTо

$$
R_{T}(s) \leqslant 2 c_{4}\left(1-\kappa_{3}\right)^{-1} f(s) \quad \forall s, \quad 0<s<s_{0}, \quad \forall t, 0<t<T\left(s_{0}\right),
$$

если выполнено условие малости

$$
\begin{aligned}
h_{0}(s) & \leqslant k_{2} s^{\frac{m(p+1)-1+\nu}{\mu}}=k_{2} s^{\alpha_{2}} \\
& \Rightarrow f(s)<c_{4}\left(\psi_{1} \chi_{1}\right)^{(1+\mu)(\nu-1) \beta_{3}} k^{\mu \beta_{3}(1+\mu)} s \quad \forall s, 0<s<s_{2},
\end{aligned}
$$

где $s_{2}=2^{-1}\left(1-\kappa_{3}\right) s_{0}$, а $k_{2}$ удовлетворяет условию

$$
\begin{aligned}
(2(1 & \left.\left.-\kappa_{3}\right)^{-1} c_{4}\left(\psi_{1} \chi_{1}\right)^{\nu-1} k_{2}^{\mu}\right)^{(1+\mu) \beta_{3}} \\
& +\left(2\left(1-\kappa_{3}\right)^{-1} c_{4}\left(\psi_{1} \chi_{1}\right)^{\nu-1} k_{2}^{\mu}\right)^{\left(1-\beta_{4}\right)(1+\mu) \beta_{3}}<2^{-1}\left(1-\kappa_{3}\right) .
\end{aligned}
$$

Тем самым, доказано наличие ЗРН-эффекта при условии (2.25).

Оценка (2.16) при $q<p$ выведена в теореме 1. Оценим теперь левый фронт иначе. Из (4.35), очевидно, следует, что

$$
R_{T}\left(s-R_{T}(s)\right) \leqslant \kappa_{3} R_{T}(s) \quad \forall s<0, \quad \kappa_{3}=2^{-\mu \beta_{3}}<1,
$$


откуда в силу леммы 8.1

$$
\eta_{-}(T) \geqslant-R_{T}(0) \quad \forall T \geqslant 0 .
$$

Оценим $R_{T}(0)$ сверху. Из (4.35) в силу леммы 8.2 из дополнения вытекает, что

$$
\begin{aligned}
R_{T}(0) \leqslant c & \left(\chi_{1}^{-(1+\mu)(1-\nu) \beta_{3}}\left(\widetilde{s}_{1}\left(\psi_{1} \chi_{1}\right)^{1-\nu} h\left(\widetilde{s}_{1}\left(\psi_{1} \chi_{1}\right)\right)^{1+\mu}\right)^{\mu \beta_{3}}\right. \\
& +\widetilde{s}_{1}\left(\psi_{1} \chi_{1}\right)^{\beta_{4}}\left(\psi_{1} \chi_{1}\right)^{-(1+\mu)(1-\nu)\left(1-\beta_{4}\right) \beta_{3}} \\
& \left.\times\left(\widetilde{s}_{1}\left(\psi_{1} \chi_{1}\right)^{1-\nu} h\left(\widetilde{s}_{1}\left(\psi_{1} \chi_{1}\right)\right)^{1+\mu}\right)^{\mu \beta_{3}\left(1-\beta_{4}\right)}\right),
\end{aligned}
$$

где $\widetilde{s}_{1}\left(\psi_{1} \chi_{1}\right) \geqslant 0$ - максимальный корень уравнения

$$
\begin{aligned}
& c_{4}\left(\psi_{1} \chi_{1}\right)^{-(1+\mu)(1-\nu) \beta_{3}}\left(s^{1-\nu} h(s)^{1+\mu}\right)^{\mu \beta_{3}} \\
& \quad+s^{\beta_{4}}\left(c_{4}\left(\psi_{1} \chi_{1}\right)^{-(1+\mu)(1-\nu) \beta_{3}}\left(s^{1-\nu} h(s)^{1+\mu}\right)^{\mu \beta_{3}}\right)^{1-\beta_{4}}=2^{-1}\left(1-\kappa_{3}\right) s .
\end{aligned}
$$

Оценивая правую часть неравенства (4.40) с помощью (4.41), приходим к соотношению

$$
\eta_{-}(t) \geqslant-c \widetilde{s}_{1}\left(\psi_{1} \chi_{1}\right) \quad \forall t>0 .
$$

Справедливость оценки (2.24), очевидно, вытекает из соотношений (2.16) и (4.42) в силу правильной монотонности функции $h(s)$. Теорема доказана.

ДокАЗАТЕЛЬСтво теоРемы 4. Докажем сначала возникновение ЗРН-эффекта при выполнении условия малости (2.25). В силу неравенства Гёльдера имеем, что

$$
\begin{aligned}
I_{T}(s-\delta) & \leqslant E_{T}(s-\delta)^{\frac{p-\lambda}{q-\lambda}} J_{T}(s-\delta)^{\frac{q-p}{q-\lambda}} \\
& \leqslant \Psi_{0}^{T}(s-\delta, \xi)^{\frac{p-\lambda}{q-\lambda}} J_{T}(s-\delta)^{\frac{q-p}{q-\lambda}} H_{T}(s-\delta)^{\frac{p-\lambda}{q-\lambda}(1-\xi)}
\end{aligned}
$$

$\forall s \in \mathbb{R}^{1}, \forall \delta>0,0<\xi<1$. Оценивая правую часть (4.43) с помощью неравенства (4.30) и применяя последовательно для оценки правой части полученного выражения соотношения (4.30), (4.32) и (3.5), приходим к (4.33). Используя монотонность и непрерывность функции $I_{T}(s)$ по $s$ и $T$ и повторяя далее рассуждения, содержащиеся в предыдущей теореме, приходим к требуемому. Так же, как и в теореме 3, выводится и оценка (4.42).

Оценим левый фронт в случае $\lambda<p<q$. Из (3.5) при $\Omega(s)=\Omega(\Lambda, y, s)$ в силу неравенства Юнга с $\varepsilon$ и соотношения (4.14) вытекает, что

$$
T^{-1} E_{T}(s) \leqslant \varepsilon T^{-1} E_{T}(s+\delta)+c(\varepsilon) T^{\frac{q+1}{q-p}} \delta^{\frac{m(p+1)(q+1)}{q-p}}\left(-\eta_{-}(T)\right)^{n}
$$

$\forall \delta>0, s+\delta<0, \forall \varepsilon>0$. Полагая в предыдущем неравенстве

$$
\varepsilon=2^{-\frac{m(p+1)(q+1)}{q-p}-1}
$$

и применяя к нему итеративную процедуру, аналогичную содержащейся в доказательстве леммы 3.3, получаем оценку

$$
T^{-1} E_{T}(s) \leqslant c T^{\frac{q+1}{q-p}}(-s)^{\frac{m(p+1)(q+1)}{q-p}}\left(-\eta_{-}(T)\right)^{n} \quad \forall s<0 .
$$

Из (4.39) в силу леммы 8.1 из дополнения вытекает, что

$$
\eta_{-}(T) \geqslant s_{0}-c\left(\chi_{1}^{-\frac{1-\nu}{\mu}} I_{T}\left(s_{0}\right)\right)^{\mu \beta_{3}} \quad \forall s_{0}<0 .
$$


Оценив правую часть (4.45) с помощью неравенства Гёльдера, с учетом (4.14) получим, что

$$
\eta_{-}(T) \geqslant s_{0}-c\left(\chi_{1}^{-\frac{1-\nu}{\mu}}\left(T\left(-\eta_{-}(T)\right)^{n}\right)^{\frac{q-p}{q+1}} E_{T}\left(s_{0}\right)^{\frac{p+1}{q+1}}\right)^{\mu \beta_{3}} \quad \forall s_{0}<0 .
$$

Применяя для оценки правой части предыдущего выражения неравенство (4.44), приходим к соотношению

$$
\eta_{-}(T) \geqslant s_{0}-c\left(\chi_{1}^{-\frac{1-\nu}{\mu}} T^{q-1} q\left(-\eta_{-}(T)\right)^{n}\left(-s_{0}\right)^{-\frac{m(p+1)^{2}}{q-p}}\right)^{\mu \beta_{3}} \equiv \phi\left(s_{0}\right) \quad \forall s_{0}<0 .
$$

Оптимизируя функцию $\phi(s)$, получаем, что

$$
\begin{gathered}
\max _{-\infty<s_{0}<0} \phi\left(s_{0}\right)=\phi\left(s_{\mathrm{opt}}\right), \quad s_{\mathrm{opt}}=-c T^{\gamma_{4}}\left|\eta_{-}(T)\right|^{\gamma_{5}} \chi_{1}^{-\gamma_{6}}, \\
\gamma_{4}=\frac{\mu(q+1)}{m(p+1)(q+1) \mu+(q-p)(m(p+1)-1+\nu)}, \\
\gamma_{5}=\frac{n(q-p)}{q+1} \gamma_{4}, \quad \gamma_{6}=\frac{(1-\nu)(q-p)}{\mu(q+1)} \gamma_{4},
\end{gathered}
$$

откуда вытекает следующее неравенство:

$$
\eta_{-}(T) \geqslant-c \chi_{1}^{-\frac{\gamma_{6}}{1-\gamma_{5}}} T^{\frac{\gamma_{4}}{1-\gamma_{5}}}=-c\left(\chi_{1}^{-\frac{q-p}{p-\lambda}} T\right)^{\beta_{2}} \quad \forall T>0 .
$$

Вывод оценки (4.46) в случае $\lambda<p=q$ содержится в доказательстве теоремы 5 .

Справедливость (2.27), очевидно, вытекает из оценок (4.42), (4.46) в силу правильной монотонности $h(s)$. Теорема 4 доказана.

\section{§ 5. Обратное движение левого фронта}

ДокАЗАТЕЛЬСтво теоремы 5. Рассмотрим сначала случай $\lambda<q<p$. Интегрируя (4.1) по $t$ и применяя затем неравенство Гёльдера, приходим к соотношению

$$
I_{T}(s-\delta, \tau-\omega) \leqslant c \Psi_{T-\tau+\omega}^{T}\left(s-\delta, \frac{p+1}{q+1}\right)^{1-\theta} F_{T}(s-\delta, \tau-\omega)^{\theta}
$$

$\forall s \in \mathbb{R}^{1}, \forall \delta>0$. Возводя обе части неравенства (4.29) в степень $\xi$, интегрируя по $t$ и применяя затем неравенство Гёльдера, получаем, что

$\Psi_{T-\tau+\omega}^{T}(s-\delta, \xi) \leqslant c F_{T}(s-\delta, \tau-\omega)^{\nu_{1}} J_{T}(s-\delta, \tau-\omega)^{1-\nu_{1}} \quad \forall s \in \mathbb{R}^{1}, \quad \forall \delta>0$.

В силу леммы 3.4 также имеем, что

$$
\Psi_{T-\tau+\omega}^{T}\left(s-\delta, \frac{p+1}{q+1}\right) \leqslant c \Psi_{T-\tau}^{T}(s, \xi) R_{T}(s, \delta, \tau, \omega)^{\frac{p+1}{q+1}-\xi} .
$$

Оценим правую часть (5.3) с помощью неравенства (5.2), а полученное соотношение применим для оценки первого сомножителя в (5.1). В результате имеем, что

$I_{T}(s-\delta, \tau-\omega) \leqslant c R_{T}(s+\delta, \delta, \tau+\omega, \omega)^{\mu} F(s, \tau)^{\nu} J_{T}(s, \tau)^{1-\nu} \quad \forall s \in \mathbb{R}^{1}, \quad \forall \delta>0$, 
$\forall \tau, \omega, 0<\omega<\tau<T, \mu, \nu-$ из (2.23). Полагая в (3.7) $s=s_{0}, s_{i}=s_{i-1}-\delta$, $i=1,2, \ldots$, и суммируя полученные неравенства, приходим к следующему соотношению:

$$
\begin{aligned}
J_{T}(s-\delta, \tau-\omega) & \leqslant c\left(\psi_{1} \chi_{1}\right)^{-1} \delta \sum_{i=0}^{\infty}\left(\delta^{-m(p+1)} I_{T}(s-i \delta, \tau)+\omega^{-1} E_{T}(s-i \delta, \tau)\right) \\
& \leqslant c\left(\psi_{1} \chi_{1}\right)^{-1} \int_{-\infty}^{s+\delta}\left(\delta^{-m(p+1)} I_{T}(\theta, \tau)+\omega^{-1} E_{T}(\theta, \tau)\right) d \theta .
\end{aligned}
$$

Из (5.5), очевидно, вытекает неравенство

$$
J_{T}(s-\delta, \tau-\omega) \leqslant c\left(\psi_{1} \chi_{1}\right)^{-1} \int_{-\infty}^{s}\left(\delta^{-m(p+1)} I_{T}(\theta, \tau)+\omega^{-1} E_{T}(\theta, \tau)\right) d \theta .
$$

В теоремах 3,4 показано, что при выполнении условия малости $(2.25) I_{T}(0)=$ $E_{T}(0)=0 \forall T, 0<T<T^{*}\left(s_{0}\right)$, поэтому в силу монотонности функций $I_{T}(s, \tau)$ и $E_{T}(s, \tau)$ из $(5.6)$, очевидно, следует соотношение

$$
\begin{gathered}
J_{T}(s-\delta, \tau-\omega) \leqslant c\left(\psi_{1} \chi_{1}\right)^{-1} s\left(\delta^{-m(p+1)} I_{T}(s, \tau)+\omega^{-1} E_{T}(s, \tau)\right) \\
\forall s, \quad 0<s<s_{0}, \quad \forall \delta>0,
\end{gathered}
$$

$\forall \tau, \omega, 0<\omega<\tau<T<T^{*}$. Используя теперь для оценки правой части (5.4) неравенства (3.7) и (5.7), после замены в полученном неравенстве аргументов приходим к соотношению

$$
I_{T}(s-\delta, \tau-\omega) \leqslant c\left(\left(\psi_{1} \chi_{1}\right)^{-1} s\right)^{1-\nu}\left(\delta^{-m(p+1)} I_{T}(s, \tau)+\omega^{-1} E_{T}(s, \tau)\right)^{1+\mu}
$$

$\forall s, 0<s<s_{0}, \forall \delta>0, \forall \tau, \omega, 0<\omega<\tau<T<T^{*}$.

Для функции $E_{T}(s, \tau)$ установим теперь соотношение, аналогичное неравенству (5.8). Положив в (3.11) $h_{2}=1, h_{1}=\xi$, получим, что

$$
E_{T}(s-\delta, \tau-\omega) \equiv \Psi_{T-\tau+\omega}^{T}(s-\delta, 1) \leqslant \Psi_{T-\tau}^{T}(s, \xi) R_{T}(s, \delta, \tau, \omega)^{1-\xi}
$$

$\forall s, 0<s<s_{0}, \forall \delta>0, \forall \tau, \omega, 0<\omega<\tau<T<T^{*}$. Оценим первый сомножитель в правой части (5.9) с помощью неравенства (5.2). В результате имеем, что

$$
E_{T}(s-\delta, \tau-\omega) \leqslant c F_{T}(s, \tau)^{\nu_{1}} J_{T}(s, \tau)^{1-\nu_{1}} R_{T}(s, \delta, \tau, \omega)^{1-\xi} .
$$

Оценивая теперь правую часть (5.10) с помощью (3.7) и (5.7), после замены в полученном неравенстве аргументов приходим к соотношению

$$
E_{T}(s-\delta, \tau-\omega) \leqslant c\left(\left(\psi_{1} \chi_{1}\right)^{-1} s\right)^{1-\nu_{1}}\left(\delta^{-m(p+1)} I_{T}(s, \tau)+\omega^{-1} E_{T}(s, \tau)\right)^{1+\mu_{1}}
$$

$\forall s, 0<s<s_{0}, \forall \delta>0, \forall \tau, \omega, 0<\omega<\tau<T<T^{*}$, где $\nu_{1}, \mu_{1}-$ из (2.31).

Выведем теперь соотношение (5.8) для случая $\lambda<p \leqslant q$. В силу неравенства Гёльдера

$$
I_{T}(s-\delta, \tau-\omega) \leqslant E_{T}(s-\delta, \tau-\omega)^{\frac{p-\lambda}{q-\lambda}} J_{T}(s-\delta, \tau-\omega)^{\frac{q-p}{q-\lambda}} .
$$

Используя для оценки первого сомножителя в правой части (5.12) неравенство (3.11) при $h_{2}=1, h_{1}=\xi$ и оценивая правую часть полученного соотношения с помощью (3.7) и (5.7), приходим к (5.8). 
Возведем теперь $(5.8)$ в степень $1+\mu_{1}$ и умножим на $\left(\left(\psi_{1} \chi_{1}\right)^{-1} s\right)^{\left(1-\nu_{1}\right)(1+\mu)}$, а $(5.11)$ - в степень $1+\mu$ и умножим на $\left(\left(\psi_{1} \chi_{1}\right)^{-1} s\right)^{(1-\nu)\left(1+\mu_{1}\right)}$. Складывая полученные неравенства, приходим к следующему соотношению:

$$
\begin{aligned}
N_{T}(s-\delta, & \tau-\omega) \equiv\left(\left(\psi_{1} \chi_{1}\right)^{-1} s\right)^{\left(1-\nu_{1}\right)(1+\mu)} I_{T}(s-\delta, \tau-\omega)^{1+\mu_{1}} \\
& +\left(\left(\psi_{1} \chi_{1}\right)^{-1} s\right)^{(1-\nu)\left(1+\mu_{1}\right)} E_{T}(s-\delta, \tau-\omega)^{1+\mu} \\
\leqslant c & \left(\left(\psi_{1} \chi_{1}\right)^{-1} s\right)^{\left(1-\nu_{1}\right)(1+\mu)+(1-\nu)\left(1+\mu_{1}\right)} \\
& \times\left(\delta^{-m(p+1)(1+\mu)\left(1+\mu_{1}\right)} I_{T}(s, \tau)^{(1+\mu)\left(1+\mu_{1}\right)}\right. \\
& \left.+\omega^{-(1+\mu)\left(1+\mu_{1}\right)} E_{T}(s, \tau)^{(1+\mu)\left(1+\mu_{1}\right)}\right)
\end{aligned}
$$

$\forall s, 0<s<s_{0}, \forall \delta>0, \forall \tau, \omega, 0<\omega<\tau<T<T^{*}$. Определим теперь аргументы $\delta, \omega$ следующим образом:

$$
\begin{gathered}
\delta=\delta\left(s, \tau, \psi_{1} \chi_{1}\right)=c_{5}\left(\left(\psi_{1} \chi_{1}\right)^{-1} s\right)^{\frac{1-\nu}{m(p+1)(1+\mu)}} I_{T}(s, \tau)^{\frac{\mu}{m(p+1)(1+\mu)}}, \\
\omega=\omega\left(s, \tau, \psi_{1} \chi_{1}\right)=c_{6}\left(\left(\psi_{1} \chi_{1}\right)^{-1} s\right)^{\frac{1-\nu_{1}}{1+\mu_{1}}} E_{T}(s, \tau)^{\frac{\mu_{1}}{1+\mu_{1}}}, \\
c_{5}=(4 c)^{\frac{1}{m(p+1)(1+\mu)\left(1+\mu_{1}\right)}}, \quad c_{6}=c_{5}^{m(p+1)},
\end{gathered}
$$

$c=$ const - из (5.13). Учитывая монотонность $I_{T}(s, \tau)$ и $E_{T}(s, \tau)$ по обоим аргументам, из (5.13) получаем следующее соотношение:

$N_{T}\left(s-c_{5}\left(\left(\psi_{1} \chi_{1}\right)^{-1} s\right)^{\gamma_{7}} N_{T}(s, \tau)^{\rho_{1}}, \tau-c_{6}\left(\left(\psi_{1} \chi_{1}\right)^{-1} s\right)^{\gamma_{8}} N_{T}(s, \tau)^{\rho_{2}}\right) \leqslant 2^{-1} N_{T}(s, \tau)$, где

$$
\begin{aligned}
\gamma_{7} & =\frac{1-\nu}{m(p+1)(1+\mu)}-\frac{\mu\left(1-\nu_{1}\right)}{m(p+1)\left(1+\mu_{1}\right)}, & \gamma_{8} & =\frac{1-\nu_{1}}{1+\mu_{1}}-\frac{\mu_{1}(1-\nu)}{1+\mu}, \\
\rho_{1} & =\frac{\mu}{m(p+1)(1+\mu)\left(1+\mu_{1}\right)}, & \rho_{2} & =\frac{\mu_{1}}{(1+\mu)\left(1+\mu_{1}\right)} .
\end{aligned}
$$

Из последнего неравенства в силу леммы 8.1 из дополнения вытекает, что

$$
\begin{array}{r}
N_{T}(s, T)=0 \quad \forall(s, T) \in\left\{s \leqslant s^{\prime}-c_{7}\left(\left(\psi_{1} \chi_{1}\right)^{-1} s^{\prime}\right)^{\gamma_{7}} N_{T}\left(s^{\prime}, T^{\prime}\right)^{\rho_{1}},\right. \\
\left.T^{*} \geqslant T \geqslant c_{8}\left(\left(\psi_{1} \chi_{1}\right)^{-1} s^{\prime}\right)^{\gamma_{8}} N_{T}\left(s^{\prime}, T^{\prime}\right)^{\rho_{2}}\right\},
\end{array}
$$

$c_{7}=c_{5}\left(1-2^{-\rho_{1}}\right)^{-1}, c_{8}=c_{6}\left(1-2^{-\rho_{2}}\right)^{-1}$.

Оценим теперь $N_{T}(s, T)$ сверху. Полагая в выражении $(3.5) s=s_{0}, s_{i}=$ $s_{i-1}-\delta / 4, i=1,2, \ldots$, складывая полученные неравенства и производя вычисления, аналогичные проведенным при выводе соотношение (5.7), приходим к соотношению

$$
\begin{gathered}
J_{T}(s-\delta) \leqslant c\left(\psi_{1} \chi_{1}\right)^{-1} s\left(\delta^{-m(p+1)} I_{T}(s)+h(s)\right) \\
\forall \delta>0, \quad \forall s, 0<s<s_{0}, \quad \forall T<T^{*} .
\end{gathered}
$$

Оценим $E_{T}(s)$ :

$$
E_{T}(s) \leqslant \Psi_{0}^{T}(s, \xi) H_{T}(s)^{1-\xi} \quad \forall s>0, \quad \forall T<T^{*} .
$$

Применяя для оценки правой части (5.16) неравенство (4.30), а затем соотношения (5.15) и (3.5), после замены в полученном выражении аргументов приходим к соотношению

$$
E_{T}(s-\delta) \leqslant c\left(\left(\psi_{1} \chi_{1}\right)^{-1} s\right)^{1-\nu_{1}}\left(\delta^{-m(p+1)} I_{T}(s)+h_{0}(s)\right)^{1+\mu_{1}}
$$


$\forall s, 0<s<s_{0}, \forall \delta>0, \forall T<T^{*}$. Из (4.37), очевидно, следует, что

$$
I_{T}(s) \leqslant c\left(\left(\psi_{1} \chi_{1}\right)^{-1} s\right)^{1-\nu} h(s)^{1+\mu} \quad \forall s, 0<s<s_{2}<s_{0},
$$

если выполнено соотношение (4.38). Положив в (5.17)

$$
\delta=\delta\left(s, T, \psi_{1} \chi_{1}\right) \equiv\left(\left(\left(\psi_{1} \chi_{1}\right)^{-1} s\right)^{1-\nu} I_{T}(s)^{\mu}\right)^{\frac{1}{m(p+1)(1+\mu)}},
$$

после вычислений с использованием оценки (5.18) приходим к соотношению

$$
E_{T}\left(s-c_{9}\left(\left(\psi_{1} \chi_{1}\right)^{-1} s\right)^{\frac{1-\nu}{m(p+1)}} h(s)^{\frac{\mu}{m(p+1)}}\right) \leqslant c\left(\left(\psi_{1} \chi_{1}\right)^{-1} s\right)^{1-\nu_{1}} h(s)^{1+\mu_{1}}
$$

$\forall s, 0<s<s_{2}, \forall T<T^{*}$, откуда в силу (2.29) вытекает неравенство

$$
E_{T}\left(s\left(1-c_{9}\left(\psi_{1} \chi_{1}\right)^{\frac{\nu-1}{m(p+1)}} k_{3}^{\frac{\mu}{m(p+1)}}\right)\right) \leqslant c\left(\left(\psi_{1} \chi_{1}\right)^{-1} s\right)^{1-\nu_{1}} h(s)^{1+\mu_{1}}
$$

$\forall s, 0<s<s_{2}, \forall T<T^{*}$. Потребуем, чтобы $k_{3}$ удовлетворяло условию

$$
k_{3}<\left(\left(2 c_{9}\right)^{-1}\left(\psi_{1} \chi_{1}\right)^{\frac{1-\nu}{m(p+1)}}\right)^{\frac{m(p+1)}{\mu}} .
$$

Из (5.19) в силу предыдущего неравенства имеем

$$
E_{T}\left(2^{-1} s\right) \leqslant c\left(\left(\psi_{1} \chi_{1}\right)^{-1} s\right)^{1-\nu_{1}} h(s)^{1+\mu_{1}} \quad \forall s, 0<s<s_{2},
$$

откуда в силу правильной монотонности функции $h(s)$ вытекает оценка

$$
E_{T}(s) \leqslant c\left(\left(\psi_{1} \chi_{1}\right)^{-1} s\right)^{1-\nu_{1}} h(s)^{1+\mu_{1}} \quad \forall s<2^{-1} s_{2} .
$$

Вспомнив определение функции $N_{T}(s, T)$, подставим оценки (5.18) и $(5.20)$ в выражение (5.14). В результате придем к следующему соотношению:

$$
\begin{gathered}
N_{T}(s, T)=0 \quad \forall(s, T) \in\left\{s \leqslant s^{\prime}-c_{10}\left(\left(\psi_{1} \chi_{1}\right)^{-1} s^{\prime}\right)^{\frac{1-\nu}{m(p+1)}} h\left(s^{\prime}\right)^{\frac{\mu}{m(p+1)}},\right. \\
\left.T^{*} \geqslant T \geqslant c\left(\left(\psi_{1} \chi_{1}\right)^{-1} s^{\prime}\right)^{1-\nu_{1}} h(s)^{\mu_{1}}\right\}
\end{gathered}
$$

$\forall s, 0<s<2^{-1} s_{2}$. Потребуем теперь, чтобы $k_{3}$ удовлетворяло также условию

$$
1-c_{10}\left(\left(\psi_{1} \chi_{1}\right)^{\nu-1} k_{3}^{\mu}\right)^{\frac{1}{m(p+1)}}=\omega\left(k_{3}\right)>0 .
$$

Из (5.21) в силу предыдущего неравенства имеем

$$
\begin{aligned}
N_{T}(s, T)=0 \quad \forall(s, T) \in\{ & s \leqslant \omega\left(k_{3}\right) s^{\prime}, \\
& \left.T^{*} \geqslant T \geqslant c\left(\left(\psi_{1} \chi_{1}\right)^{-1} s^{\prime}\right)^{1-\nu_{1}} h\left(s^{\prime}\right)^{\mu_{1}}\right\}
\end{aligned}
$$

$\forall s, 0<s<2^{-1} s_{2}$, откуда вытекает оценка (2.30).

Покажем справедливость (2.27) в случае $\lambda<p=q$. Полагая в неравенстве $(3.5) \delta=s / 2$ и устремляя в полученном соотношении $s \rightarrow \infty$, приходим к оценке

$$
I_{T}(s)=E_{T}(s) \leqslant c T h_{0}(\infty) \quad \forall s<\infty, \quad \forall T>0 .
$$

Оценивая функцию $N_{T}(s, T)$ с помощью последнего неравенства, приходим к соотношению

$$
N_{T}(s, T) \leqslant\left(c_{11} T\right)^{1+\mu}\left(\left(\psi_{1} \chi_{1}\right)^{-1} s\right)^{(1+\mu)(1-\nu)} h_{0}(\infty)^{1+\mu} \quad \forall s<\infty, \quad \forall T>0 .
$$


Полагая в предыдущем выражении

$$
s=s^{\prime}(T)=\left(\psi_{1} \chi_{1}\right)\left(c_{8}^{-(1+\mu)}\left(c_{11} h_{0}(\infty)\right)^{-\mu} T\right)^{(1-\nu)^{-1}}
$$

и подставляя полученную оценку в (5.14), приходим к неравенству

$$
\eta_{-}(T) \geqslant c h_{0}(\infty)^{-\frac{p-\lambda}{p+1}} \psi_{1} \chi_{1} T^{\beta_{5}}-c T^{\frac{1}{m(p+1)}} \quad \forall T>0,
$$

откуда вытекает (2.27).

Докажем справедливость оценки (2.32). Используя теоремы 3,4 и повторяя рассуждения, аналогичные проведенным при выводе соотношения (5.22), получаем, что

$$
\begin{gathered}
N_{T}(s, T)=0 \quad \forall(s, T) \in\left\{s \leqslant c s^{\prime}, T \geqslant c\left(\left(\psi_{1} \chi_{1}\right)^{-1} s^{\prime}\right)^{1-\nu_{1}} h(\infty)^{\mu_{1}}, s^{\prime}>s_{1}\left(\psi_{1} \chi_{1}\right)\right\}, \\
s_{1}\left(\psi_{1} \chi_{1}\right) \text { - из }(2.22), \text { откуда, очевидно, вытекает }(2.32) . \text { Теорема доказана. }
\end{gathered}
$$

\section{§6. Прямое движение правого фронта}

ДОКАЗАТЕЛЬСтво тЕОРЕмЫ 6. Интегрируя (4.25) по $t$ и применяя неравенство Гёльдера, приходим к соотношению

$$
\begin{gathered}
J_{T}(s-\delta) \leqslant c F_{T}(s-\delta)^{\sigma} \Psi_{0}^{T}\left(s-\delta, \sigma_{1}\right)^{1-\sigma} \quad \forall s \in \mathbb{R}^{1}, \quad \forall \delta>0, \\
1>\sigma=\frac{n(\lambda-q)}{n(p-q)+m(p+1)(q+1)}, \quad \sigma_{1}=\frac{m(p+1)(\lambda+1)+n(p-\lambda)}{m(p+1)(q+1)+n(p-\lambda)},
\end{gathered}
$$

откуда, очевидно, вытекает неравенство

$$
J_{T}(s-\delta) \leqslant c T^{1-\sigma} F_{T}(s-\delta)^{\sigma} H_{T}(s-\delta)^{\sigma_{1}(1-\sigma)} \quad \forall s \in \mathbb{R}^{1}, \quad \forall \delta>0 .
$$

Оценивая правую часть предыдущего соотношения с помощью (3.6), получаем, что

$$
\begin{gathered}
J_{T}(s-\delta) \leqslant c T^{1-\sigma}\left(\delta^{-m(p+1)} \Delta I_{T}(s)-\psi_{1} \chi_{1} \delta^{-1} \Delta J_{T}(s)+h_{0}(s)\right)^{1+\beta_{6}} \\
\forall s \in \mathbb{R}^{1}, \quad \forall \delta>0,
\end{gathered}
$$

$\beta_{6}$ - из (2.33). Оценивая правую часть (4.3) с помощью соотношения (3.6), получаем, что

$$
I_{T}(s-\delta) \leqslant c T^{1-\theta}\left(\delta^{-m(p+1)} \Delta I_{T}(s)-\psi_{1} \chi_{1} \delta^{-1} \Delta J_{T}(s)+h_{0}(s)\right)^{1+\alpha}
$$

$\forall s \in \mathbb{R}^{1}, \forall \delta>0$. Возводя (6.1) в степень $1+\alpha$ и умножая на $T^{(1-\theta)\left(1+\beta_{6}\right)}$, a $(6.2)$ - в степень $1+\beta_{6}$ и умножая на $T^{(1-\sigma)(1+\alpha)}$, приходим к соотношениям

$$
\begin{aligned}
A_{T}(s-\delta) \leqslant c & T^{d} \Delta A_{T}(s)^{1+\alpha} \delta^{-m(p+1)(1+\alpha)\left(1+\beta_{6}\right)} \\
& \left.+T^{l} \Delta B_{T}(s)^{1+\beta_{6}}\left(-\psi_{1} \chi_{1} \delta^{-1}\right)^{(1+\alpha)\left(1+\beta_{6}\right)}\right) \\
& +c T^{(1-\sigma)(1+\alpha)+(1-\theta)\left(1+\beta_{6}\right)} h_{0}(s)^{(1+\alpha)\left(1+\beta_{6}\right)} \\
B_{T}(s-\delta) \leqslant c & T^{d} \Delta A_{T}(s)^{1+\alpha} \delta^{-m(p+1)(1+\alpha)\left(1+\beta_{6}\right)} \\
& \left.+T^{l} \Delta B_{T}(s)^{1+\beta_{6}}\left(-\psi_{1} \chi_{1} \delta^{-1}\right)^{(1+\alpha)\left(1+\beta_{6}\right)}\right) \\
& +c T^{(1-\sigma)(1+\alpha)+(1-\theta)\left(1+\beta_{6}\right)} h_{0}(s)^{(1+\alpha)\left(1+\beta_{6}\right)}
\end{aligned}
$$


$\forall s \in \mathbb{R}^{1}, \forall \delta>0$,

$$
\begin{aligned}
A_{T}(s)=T^{(1-\sigma)(1+\alpha)} I_{T}(s)^{1+\beta_{6}}, & B_{T}(s)=T^{(1-\theta)\left(1+\beta_{6}\right)} J_{T}(s)^{1+\alpha}, \\
d=(1-\theta)\left(1+\beta_{6}\right)-\alpha(1-\sigma)(1+\alpha), & l=(1-\sigma)(1+\alpha)-\beta_{6}(1-\theta)\left(1+\beta_{6}\right), \\
\Delta A_{T}(s)=A_{T}(s)-A_{T}(s-\delta), & \Delta B_{T}(s)=B_{T}(s)-B_{T}(s-\delta) .
\end{aligned}
$$

Складывая (6.3) и (6.4), получаем, что

$$
\begin{aligned}
L_{T}(s-\delta) \equiv & A_{T}(s-\delta)+B_{T}(s-\delta) \\
\leqslant & c \Delta L_{T}(s)\left(T^{d} L_{T}(s)^{\alpha} \delta^{-m(p+1)(1+\alpha)\left(1+\beta_{6}\right)}\right. \\
& \left.+T^{l} L_{T}(s)^{\beta_{6}}\left(-\psi_{1} \chi_{1} \delta^{-1}\right)^{(1+\alpha)\left(1+\beta_{6}\right)}\right) \\
& +c T^{(1-\sigma)(1+\alpha)+(1-\theta)\left(1+\beta_{6}\right)} h(s)^{(1+\alpha)\left(1+\beta_{6}\right)}
\end{aligned}
$$

$\forall s \in \mathbb{R}^{1}, \forall \delta>0, \Delta L_{T}(s)=L_{T}(s)-L_{T}(s-\delta)$. Положим в $(6.5)$

$$
\begin{gathered}
\delta=\delta(s, T)=T^{\frac{d}{m(p+1)(1+\alpha)\left(1+\beta_{6}\right)}} L_{T}(s)^{\frac{\alpha}{m(p+1)(1+\alpha)\left(1+\beta_{6}\right)}} \\
-\psi_{1} \chi_{1} T^{\frac{l}{(1+\alpha)\left(1+\beta_{6}\right)}} L_{T}(s)^{\frac{\beta_{6}}{(1+\alpha)\left(1+\beta_{6}\right)}} \equiv Q_{T}(s) .
\end{gathered}
$$

Отсюда после очевидных преобразований приходим к следующему соотношению:

$L_{T}\left(s-Q_{T}(s)\right) \leqslant \kappa_{4}\left(L_{T}(s)+T^{(1-\sigma)(1+\alpha)+(1-\theta)\left(1+\beta_{6}\right)} h_{0}(s)^{(1+\alpha)\left(1+\beta_{6}\right)}\right) \quad \forall s \in \mathbb{R}^{1}$,

$\kappa_{4}=c(1+c)^{-1}<1, c-$ из (6.5). Возведем (6.6) в степень

$$
\frac{\alpha}{m(p+1)(1+\alpha)\left(1+\beta_{6}\right)}
$$

и умножим на $T^{\frac{d}{m(p+1)(1+\alpha)\left(1+\beta_{6}\right)}}$, затем возведем $(6.6)$ в степень

$$
\frac{\beta}{(1+\alpha)\left(1+\beta_{6}\right)}
$$

и умножим на $-\psi_{1} \chi_{1} T^{\frac{l}{(1+\alpha)\left(1+\beta_{6}\right)}}$. Сложив полученные неравенства, придем к следующему соотношению:

$$
Q_{T}\left(s-Q_{T}(s)\right) \leqslant \kappa_{5}\left(Q_{T}(s)-\psi_{1} \chi_{1} T^{1-\sigma} h_{0}(s)^{\beta_{6}}+T^{\frac{1-\theta}{m(p+1)}} h_{0}(s)^{\frac{\alpha}{m(p+1)}}\right)
$$

$\forall s \in \mathbb{R}^{1}, 0<\kappa_{5}<1$. В силу леммы 8.1 из дополнения из (6.7) вытекает соотношение

$$
Q_{T}(s)=0 \quad \forall s \leqslant s_{0}-\left(1-\kappa_{5}\right)^{-1} Q_{T}\left(s_{0}\right), \quad \forall s_{0} \leqslant 0 .
$$

Оценим $Q_{T}\left(s_{0}\right)$ сверху. Положив в $(6.5) \delta=2^{-1} s$ и устремив в полученном неравенстве $s \rightarrow \infty$, придем к соотношению

$$
L_{T}\left(s_{0}\right) \leqslant c T^{(1-\sigma)(1+\alpha)+(1-\theta)\left(1+\beta_{6}\right)} h(\infty)^{(1+\alpha)\left(1+\beta_{6}\right)} \quad \forall s_{0} \in \mathbb{R}^{1},
$$

следовательно, для $Q_{T}(s)$ выполнено

$$
Q_{T}\left(s_{0}\right) \leqslant c_{12}\left(-\psi_{1} \chi_{1} T^{1-\sigma} h(\infty)^{\beta_{6}}+T^{\frac{1-\theta}{m(p+1)}} h(\infty)^{\frac{\alpha}{m(p+1)}}\right) \quad \forall s_{0} \in \mathbb{R}^{1} .
$$


Из (6.9) в силу непрерывности $h_{0}(s)$ следует, что существует $s_{3}<\infty$ такое, что

$$
Q_{T}\left(s_{0}\right) \leqslant L \kappa_{5}\left(-\psi_{1} \chi_{1} T^{1-\sigma} h(s)^{\beta_{6}}+T^{\frac{1-\theta}{m(p+1)}} h_{0}(s)^{\frac{\alpha}{m(p+1)}}\right) \quad \forall s>s_{3},
$$

$L>\left(1-\kappa_{5}\right)^{-1}, L \kappa_{5}>c_{12}$. В силу (6.7) и леммы 8.1 из дополнения имеем

$$
\eta_{+}(T) \geqslant-\left(1-\kappa_{4}\right)^{-1} Q_{T}(0) \quad \forall T>0 .
$$

Оценим $Q_{T}(0)$ сверху. Из $(6.7),(6.10)$ в силу леммы 8.2 следует, что

$$
\begin{gathered}
Q_{T}(0) \leqslant \kappa_{5}\left(-\psi_{1} \chi_{1} T^{1-\sigma} h\left(v_{3}(T)\right)^{\beta_{6}}+T^{\frac{1-\theta}{m(p+1)}} h\left(v_{3}(T)\right)^{\frac{\alpha}{m(p+1)}}\right) \quad \forall T>0, \\
v_{3}(T)=\inf \left\{s: \kappa_{5}\left(-\psi_{1} \chi_{1} T^{1-\sigma} h(s)^{\beta_{6}}+T^{\frac{1-\theta}{m(p+1)}} h(s)^{\frac{\alpha}{m(p+1)}}\right)<\delta_{5} s\right\} \\
\delta_{5}=L^{-1}\left(1-\kappa_{5}-L^{-1}\right) .
\end{gathered}
$$

В силу предыдущего неравенства из (6.11) вытекает, что

$$
\begin{aligned}
& \eta_{+}(T) \geqslant-c \max \left\{-\psi_{1} \chi_{1} T^{1-\sigma} h\left(v_{4}(T)\right)^{\beta_{6}}, T^{\frac{1-\theta}{m(p+1)}} h\left(v_{4}(T)\right)^{\frac{\alpha}{m(p+1)}}\right\} \forall T>0,(6.12) \\
& v_{4}(T)=\max \left\{\inf \left\{s:-\psi_{1} \chi_{1} T^{1-\sigma} h(s)^{\beta_{6}}<\delta_{6} s\right\}, \inf \left\{s: T^{\frac{1-\theta}{m(p+1)}} h(s)^{\frac{\alpha}{m(p+1)}}<\delta_{6} s\right\}\right\}, \\
& \delta_{6}=\left(2 \kappa_{5}\right)^{-1} \delta_{5} . \text { Из }(6.12), \text { очевидно, следует оценка }
\end{aligned}
$$

$$
\begin{gathered}
\eta_{+}(T) \geqslant-c \max \left\{-\psi_{1} \chi_{1} T^{1-\sigma} h\left(\widetilde{v}_{2}(T)\right)^{\beta_{6}}, T^{\frac{1-\theta}{m(p+1)}} h\left(\widetilde{v}_{1}(T)\right)^{\frac{\alpha}{m(p+1)}}\right\} \quad \forall T>0,(6.13) \\
\widetilde{v}_{2}(T)=\inf \left\{s:-\psi_{1} \chi_{1} T^{1-\sigma} h(s)^{\beta_{6}}<\delta_{6} s\right\}, \\
\widetilde{v}_{1}(T)=\inf \left\{s: T^{\frac{1-\theta}{m(p+1)}} h(s)^{\frac{\alpha}{m(p+1)}}<\delta_{6} s\right\} .
\end{gathered}
$$

Соотношение (2.34) вытекает из (6.13) в силу правильной монотонности $h(s)$. Теорема 6 доказана.

\section{§ 7. Эволюция геометрии носителей}

ДокаЗАТЕЛЬСТво теОРемы 7. Пусть в (1.1) $\lambda<q, \lambda<p$ или $\lambda<p \leqslant q$,

$$
p \geqslant \frac{n q-m(q+1)}{n+m(q+1)} .
$$

Покажем, что $\operatorname{supp} u(t, \cdot) \subseteq D^{(1)}$. В силу следствия 4 имеет место включение

$$
\operatorname{supp} u(t, \cdot) \subseteq \widetilde{D}^{(1)}=\bigcap_{\psi}\left\{x:(\psi, x) \geqslant-R-C \psi_{1}^{-\frac{1-\nu}{m(p+1)-1+\nu}}\right\} \quad \forall t>0 .
$$

Очевидно, $\widetilde{D}^{(1)}$ есть тело вращения некоторой кривой вокруг оси $0 x_{1}$, которая, в свою очередь, является огибающей следующего семейства прямых, лежащих в плоскости $x_{1} 0 x_{2}$ :

$$
x_{1} \cos \phi+x_{2} \sin \phi=-R-C \cos \phi^{-\frac{1-\nu}{m(p+1)-1+\nu}}, \quad \phi \in\left[0,2^{-1} \pi\right) .
$$

Найдем эту огибающую. Дифференцируя (7.1) по $\phi$, получаем

$$
-x_{1} \sin \phi+x_{2} \cos \phi=-C_{1} \cos \phi^{-\frac{m(p+1)}{m(p+1)-1+\nu}} \sin \phi, \quad \phi \in\left[0,2^{-1} \pi\right),
$$


$C_{1}=C(1-\nu)(m(p+1)-1+\nu)^{-1}$. Решая систему $(7.1),(7.2)$, приходим к уравнению искомой огибающей: $x_{1}=\Phi_{1}(\phi), x_{2}=-\Phi_{2}(\phi), x_{3}=\cdots=x_{n}=0$, $\phi \in\left[0,2^{-1} \pi\right)$, где

$$
\begin{aligned}
& \Phi_{1}(\phi)=-R-\cos \phi^{-\frac{m(p+1)}{m(p+1)-1+\nu}}\left(C_{2} \cos ^{2} \phi-C_{1}\right), \quad C_{2}=C+C_{1}, \\
& \Phi_{2}(\phi)=\sin \phi\left(R+C_{2} \cos \phi^{-\frac{1-\nu}{m(p+1)-1+\nu}}\right) .
\end{aligned}
$$

Отсюда, очевидно, вытекает, что

$$
\widetilde{D}^{(1)}=\left\{x: x_{1} \geqslant \Phi_{1}(\phi),\left|x^{\prime}\right| \leqslant \Phi_{2}(\phi), \phi \in\left[0,2^{-1} \pi\right)\right\} .
$$

Нетрудно проверить, что $\lim _{\phi \rightarrow \pi / 2} \Phi_{1}(\phi) \Phi_{2}(\phi)^{-\frac{m(p+1)}{1-\nu}}<\infty$, следовательно, существуют постоянные $A_{6}>0, A_{7}>0$ такие, что $\widetilde{D}^{(1)} \subseteq D^{(1)}$. Тем самым, доказаны соответствующие включения в утверждениях 1)-3), 5), 7).

Пусть в (1.1) $q<\lambda<p+m(p+1)(q+1) n^{-1}, q<p$. Покажем, что $\operatorname{supp} u(t, \cdot) \subseteq D_{t}^{(2)}$. Сделав замену переменных $u(t, x)=\widetilde{u}\left(t_{1},-x_{1}, x^{\prime}\right)$, легко проверить, что $\widetilde{u}(t, x)$ удовлетворяет уравнению $(1.1)$ с $\chi=(-1,0, \ldots, 0)$, поэтому в силу теоремы 6 для носителя решения $u(t, x)$ задачи $(1.1)-(1.3)$

$$
\operatorname{supp} u(t, \cdot) \subseteq D_{t}^{(2)}=\bigcap_{\psi}\left\{x \in \mathbb{R}^{n}:(\psi, x) \leqslant R+A_{8} t^{\beta_{1}}+A_{9} \psi_{1} t^{\beta_{5}}\right\} \quad \forall t>0 .
$$

Область $D_{t}^{(2)}$ есть тело вращения вокруг оси $0 x_{1}$, огибающей семейства прямых

$$
\left(x_{1}-A_{9} t^{\beta_{5}}\right) \cos \phi+x_{2} \sin \phi=R+A_{8} t^{\beta_{1}}, \quad \phi \in\left[0,2^{-1} \pi\right], \quad x_{3}=\cdots=x_{n}=0,
$$

которые являются касательными к дуге окружности

$$
\left\{x:\left(x_{1}-A_{9} t^{\beta_{5}}\right)^{2}+\left|x_{2}\right|^{2}=\left(R+A_{8} t^{\beta_{1}}\right)^{2}, x_{1} \geqslant R+A_{9} t^{\beta_{5}}, x_{3}=\cdots=x_{n}=0\right\},
$$

а также луча

$$
\left\{x: x_{2}=R+A_{8} t^{\beta_{1}}, x_{1} \leqslant R+A_{9} t^{\beta_{5}}, x_{3}=\cdots=x_{n}=0\right\} .
$$

Отсюда, очевидно, вытекает искомое включение. Тем самым, доказаны соответствующие включения в утверждениях 1), 4), 6) теоремы 7.

Пусть в (1.1) $q<p$. Тогда в силу теорем $1-3,6$

$$
\operatorname{supp} u(t, \cdot) \subseteq \bigcap_{\psi: \psi_{1}=0}\left\{x \in \mathbb{R}^{n}:(\psi, x) \leqslant R+A_{12} t^{\beta_{1}}\right\}=D_{t}^{(5)} \quad \forall t>0 .
$$

Включения

$$
\begin{array}{lll}
\operatorname{supp} u(t, \cdot) \subseteq D_{t}^{(4)} & \text { при } & \lambda<p, \quad \lambda<q, \quad p \geqslant \frac{n q-m(q+1)}{n+m(q+1)}, \\
\operatorname{supp} u(t, \cdot) \subseteq D_{t}^{(6)} & \text { при } & q<p<\lambda, \\
\operatorname{supp} u(t, \cdot) \subseteq D_{t}^{(7)} & \text { при } & q<p=\lambda
\end{array}
$$

вытекают из теорем 5, 1, 2 соответственно. Используя неравенства (2.43), (5.23) и проводя вычисления, аналогичные представленным в выводе включения

$$
\operatorname{supp} u(t, \cdot) \subseteq D_{t}^{(2)},
$$


нетрудно также доказать справедливость, соответственно, включений

$$
\operatorname{supp} u(t, \cdot) \subseteq D_{t}^{(3)}
$$

в утверждении 2) и

$$
\operatorname{supp} u(t, \cdot) \subseteq D_{t}^{(8)}
$$

в утверждении 7). Теорема 7 доказана.

\section{§ 8. Дополнение}

Лемма 8.1. Пусть $f\left(s_{1}, s_{2}\right)$ - неотрицательная непрерывная неубывающая по каждому из своих аргументов функиия, удовлетворяющая следующему соотношению:

$$
\begin{gathered}
f\left(s_{1}-c_{1} s_{1_{+}}^{\alpha_{1}} f\left(s_{1}, s_{2}\right)^{\beta_{1}}, s_{2}-c_{2} s_{1_{+}}^{\alpha_{2}} f\left(s_{1}, s_{2}\right)^{\beta_{2}}\right) \leqslant \varepsilon f\left(s_{1}, s_{2}\right) \\
\forall s_{i}<\widetilde{s}_{i}^{(0)}, \quad i=1,2, \quad 0<\varepsilon<1, \quad 0<c_{i}<\infty \\
0 \leqslant \alpha_{i}<\infty, \quad 0<\beta_{i}<\infty, \quad i=1,2 .
\end{gathered}
$$

Тогда для $\forall s_{1}^{(0)}<\widetilde{s}_{1}^{(0)}, \forall s_{2}^{(0)}<\widetilde{s}_{2}^{(0)}$

$f\left(s_{1}, s_{2}\right)=0 \quad \forall\left(s_{1}, s_{2}\right) \in\left\{s_{i} \leqslant s_{i}^{(0)}-c_{i}\left(1-\varepsilon^{\beta_{i}}\right)^{-1}\left(s_{1_{+}}\right)^{\alpha_{i}} f\left(s_{1}^{(0)}, s_{2}^{(0)}\right)^{\beta_{i}}, i=1,2\right\}$.

ДокАЗАТЕЛЬство. Зафиксируем $s_{i}^{(0)}, i=1,2$, и построим последовательности $\left\{s_{1}^{(i)}\right\},\left\{s_{2}^{(i)}\right\}$ по следующему рекуррентному соотношению:

$$
\begin{array}{ll}
s_{1}^{(i)}=s_{1}^{(i-1)}-c_{1}\left(s_{1_{+}}^{(i-1)}\right)^{\alpha_{1}} f\left(s_{1}^{(i-1)}, s_{2}^{(i-1)}\right)^{\beta_{1}}, & i=1,2, \ldots, \\
s_{2}^{(i)}=s_{2}^{(i-1)}-c_{2}\left(s_{1_{+}}^{(i-1)}\right)^{\alpha_{2}} f\left(s_{1}^{(i-1)}, s_{2}^{(i-1)}\right)^{\beta_{2}}, & i=1,2, \ldots .
\end{array}
$$

В силу неотрицательности функции $f\left(s_{1}, s_{2}\right)$ имеем

$$
s_{1}^{(i)} \leqslant s_{1}^{(i-1)}, \quad s_{2}^{(i)} \leqslant s_{2}^{(i-1)} \quad \forall i=1,2, \ldots .
$$

Согласно неравенству (8.1) и построенному рекуррентному соотношению имеем

$$
f\left(s_{1}^{(i+1)}, s_{2}^{(i+1)}\right) \leqslant \varepsilon f\left(s_{1}^{(i)}, s_{2}^{(i)}\right) \leqslant \varepsilon^{i+1} f\left(s_{1}^{(0)}, s_{2}^{(0)}\right) .
$$

Оценим снизу $s_{1}^{(i)}, s_{2}^{(i)}, i=1,2, \ldots$. В силу (8.2) верна следующая цепочка неравенств:

$$
\begin{aligned}
s_{1}^{(i)} & =s_{1}^{(0)}-\sum_{k=0}^{i-1} c_{1}\left(s_{1_{+}}^{(k)}\right)^{\alpha_{1}} f\left(s_{1}^{(k)}, s_{2}^{(k)}\right)^{\beta_{1}} \geqslant s_{1}^{(0)}-c_{1} \sum_{k=0}^{i-1} \varepsilon^{k \beta_{1}}\left(s_{1_{+}}^{(0)}\right)^{\alpha_{1}} f\left(s_{1}^{(0)}, s_{2}^{(0)}\right)^{\beta_{1}} \\
& \geqslant s_{1}^{(0)}-c_{1}\left(1-\varepsilon^{\beta_{1}}\right)^{-1}\left(s_{1_{+}}^{(0)}\right)^{\alpha_{1}} f\left(s_{1}^{(0)}, s_{2}^{(0)}\right)^{\beta_{1}} .
\end{aligned}
$$

Аналогично получаем, что

$$
s_{2}^{(i)} \geqslant s_{2}^{(0)}-c_{2}\left(1-\varepsilon^{\beta_{2}}\right)^{-1}\left(s_{1_{+}}^{(0)}\right)^{\alpha_{2}} f\left(s_{1}^{(0)}, s_{2}^{(0)}\right)^{\beta_{2}} .
$$

Устремляя $i \rightarrow \infty$ в (8.2)-(8.4), в силу неубывания $f\left(s_{1}, s_{2}\right)$ приходим к требуемому. Лемма доказана. 
Лемма 8.2. Пусть неотрицательные непрерьвные неубывающие функции $f(s), g(s)$ удовлетворяют соотношению

$$
f\left(s-f(s)-\gamma s^{\alpha} f(s)^{1-\alpha}\right) \leqslant \varepsilon f(s)+g(s)
$$

$\forall s \geqslant 0, \gamma \geqslant 0,0<\varepsilon<1,0 \leqslant \alpha<1$.

Тогда при выполнении следующих требований:

1) существуют $s_{0}^{\prime}<\infty u k>(1-\varepsilon)^{-1}$ такие, что

$$
f(s) \leqslant k g(s) \quad \forall s>s_{0}^{\prime}>\widetilde{s}_{1} \geqslant 0 ;
$$

2) для $g(s)$ въполнено

$$
g(s) \leqslant a s \quad \forall s \geqslant \widetilde{s}_{1},
$$

где

$$
k a+\gamma(k a)^{1-\alpha}<1-\varepsilon-k^{-1}
$$

3) $g(s)$ удовлетворяет условию правильной монотонности:

$$
g\left(s\left(1-k a-\gamma(k a)^{1-\alpha}\right)\right) \geqslant\left(1-k a-\gamma(k a)^{1-\alpha}\right) g(s) \quad \forall s \geqslant \widetilde{s}_{1},
$$

имеет место следующее соотношение:

$$
f(s) \leqslant k g(s) \quad \forall s \geqslant \widetilde{s}_{1} .
$$

ДокАЗАТЕЛЬСтво. Зафиксируем произвольно $s_{0}>s_{0}^{\prime}$ и построим последовательность $\left\{s_{i}\right\}, i=1,2, \ldots$ :

$$
s_{i}=s_{i-1}-f\left(s_{i-1}\right)-\gamma s_{i}^{\alpha} f\left(s_{i-1}\right)^{1-\alpha}, \quad s_{i-1} \geqslant \widetilde{s}_{1}, \quad i=1,2, \ldots .
$$

В силу (8.6) $f\left(s_{0}\right) \leqslant k g\left(s_{0}\right)$. Предположим, что для некоторого $i \in \mathbb{N}$

$$
f\left(s_{i}\right) \leqslant k g\left(s_{i}\right), \quad s_{i} \geqslant \widetilde{s}_{1} .
$$

Докажем справедливость (8.10) для $i:=i+1$. В силу (8.5), (8.7), предположения (8.10) и соотношения (8.8) имеем, что

$$
f\left(s_{i+1}\right) \leqslant \varepsilon f\left(s_{i}\right)+g\left(s_{i}\right) \leqslant(k \varepsilon+1)\left(1-k a-\gamma(k a)^{1-\alpha}\right)^{-1} g\left(s_{i+1}\right) \leqslant k g\left(s_{i+1}\right) .
$$

Покажем теперь, что если $\widetilde{s}_{1}=0$, то $\lim _{i \rightarrow \infty} s_{i}=0$. Из неравенств (8.11) и (8.7) следует, что

$$
\begin{aligned}
s_{i+1} & =s_{i}-f\left(s_{i}\right)-\gamma s_{i_{+}}^{\alpha} f\left(s_{i}\right)^{1-\alpha} \geqslant s_{i}-k g\left(s_{i}\right)-\gamma s_{i_{+}}^{\alpha}\left(k g\left(s_{i}\right)\right)^{1-\alpha} \\
& \geqslant s_{i}\left(1-k a-\gamma(k a)^{1-\alpha}\right) \geqslant 0 .
\end{aligned}
$$

В силу монотонного невозрастания последовательность $\left\{s_{i}\right\}$ имеет предел

$$
\lim _{i \rightarrow \infty} s_{i}=\widetilde{s}
$$

Устремляя $i \rightarrow \infty$ в (8.12), получаем $f(\widetilde{s})=g(\widetilde{s})=0$, откуда $\widetilde{s}=0$. В силу произвольности выбора стартовой точки $s_{0}$ приходим к утверждению леммы.

ЛЕмма 8.3. Пусть $\Omega$ - произвольная ограниченная область в $\mathbb{R}^{n}$. Тогда если $q<p, \lambda \leqslant p+\frac{m(p+1)(q+1)}{n}$ или $\lambda<p \leqslant q$, имеет место вложение

$$
C\left([0, T] ; L_{q+1}(\Omega)\right) \cap L_{p+1}\left(0, T ; \stackrel{\circ}{W}_{p+1}^{m}(\Omega)\right) \subset L_{\lambda+1}((0, T) \times \Omega) .
$$


ДокАЗАТЕЛЬСтво. Для данной области $\Omega$ справедливо интерполяционное неравенство Гальярдо-Ниренберга вида

$$
\|u\|_{L_{\lambda+1}(\Omega)} \leqslant \widetilde{d}_{5}\left\|D_{x}^{m} u\right\|_{L_{p+1}(\Omega)}^{\widetilde{\theta}}\|u\|_{L_{q+1}(\Omega)}^{1-\widetilde{\theta}},
$$

где $u(t, x)$ - произвольная по $x$ функция из пространства $\stackrel{\circ}{W} \underset{p+1}{m}(\Omega) \cap L_{q+1}(\Omega)$,

$$
\widetilde{d}_{5}>0, \quad 0<\widetilde{\theta}=\frac{n(\lambda-q)(p+1)}{(\lambda+1)(n(p-q)+m(p+1)(q+1))}<1 .
$$

Возведя неравенство (8.14) в степень $\lambda+1$, проинтегрировав полученное соотношение по $t$ и применив неравенство Гёльдера, после очевидных преобразований придем к выражению вида

$$
\|u\|_{L_{\lambda+1}((0, T) \times \Omega)} \leqslant \widetilde{d}_{5} T^{\frac{1-\sigma}{\lambda+1}}\left\|D_{x}^{m} u\right\|_{L_{p+1}((0, T) \times \Omega)}^{\widetilde{\theta}}\|u\|_{C\left([0, T] ; L_{q+1}(\Omega)\right)}^{1-\widetilde{\theta}},
$$

$0<\sigma=\widetilde{\theta}(\lambda+1) /(p+1)<1$, откуда следует (8.13). В случае $\lambda<q, \lambda<p$ искомое вложение, очевидно, вытекает из вложений $L_{q+1}(\Omega) \subset L_{\lambda+1}(\Omega)$ и $\stackrel{\circ}{W} \underset{p+1}{m}(\Omega) \subset$ $L_{\lambda+1}(\Omega)$. Лемма доказана.

\section{Список литературы}

[1] О. А. Олейник, Калашников А. С., Чжоу Юй-линь, “Задача Коши и краевые задачи для уравнений типа нестационарной фильтрации", Изв. АН CCCP. Cер. матем., 22:5 (1958), 667-704.

[2] А.С. Калашников, "Некоторые вопросы качественной теории нелинейных вырождающихся параболических уравнений второго порядка", УМH, 42:2 (1987), 135-176.

[3] E. Di Benedetto, Degenerate parabolic equations, Springer-Verlag, New York, 1993.

[4] А.С. Калашников, "О характере распространения возмущений в процессах, описываемых квазилинейными параболическими уравнениями", Труды сем. им. И.Г. Петровского, № 1, Изд-во МГУ, М., 1975, 135-144.

[5] B. H. Gilding, L. A. Peletier, "The Cauchy problem for an equation in the theory of infiltration", Arch. Ration. Mech. Anal., 61 (1976), 127-140.

[6] J. I. Diaz, R. Kersner, "On a nonlinear degenerate parabolic equation in infiltration or evaporation through a porous medium", J. Differential Equations, 69 (1987), 368403.

[7] B. H. Gilding, "Improved theory for a nonlinear degenerate parabolic equation", Ann. Scuola Norm. Sup. Pisa Cl. Sci. (4), 16:2 (1989), 165-224.

[8] Ж.-Л. Лионс, Некоторые методы решения нелинейных краевых задач, Мир, М., 1972.

[9] Ю.А. Дубинский, "Нелинейные эллиптические и параболические уравнения", Современные проблемы математики, 9, ВИНИТИ, М., 1976, 5-130.

[10] O. Grange, F. Mignot, "Sur la résolution d'une équation et d'une inéquation paraboliques non linéaires", J. Funct. Anal., 11:1 (1972), 77-92.

[11] F. Bernis, "Existence results for doubly nonlinear higher order parabolic equations on unbounded domains", Math. Ann., 279:3 (1988), 373-394.

[12] H. W. Alt, S. Luckhaus, "Quasilinear elliptic-parabolic differential equations", Math. Z., 183 (1983), 311-341.

[13] Ph. Benilan, P. Wittbold, "On mild and weak solutions of elliptic-parabolic problems", Adv. Differential Equations, 1:6 (1996), 1053-1073. 
[14] L. Alvarez, J. I. Diaz, R. Kersner, "On the initial growth of the interfaces in nonlinear diffusion-convection processes", Nonlinear diffusion equations and their equilibrium states I, eds. W.-M. Ni, L. A. Peletier, J. Serrin, Springer-Verlag, New York, 1987, $1-20$.

[15] L. Alvarez, J. I. Diaz, "Sufficiant and necessary initial mass conditions for the existence of a waiting time in nonlinear-convection processes", J. Math. Anal. Appl., 155 (1991), 378-393.

[16] B. H. Gilding, "Properties of solutions of an equation in the theory of infiltration", Arch. Ration. Mech. Anal., 65 (1977), 203-225.

[17] R. E. Grundy, "Asymptotic solution of a model nonlinear convective diffusion eauation", IMA J. Appl. Math., 31 (1983), 121-137.

[18] Ph. Laurencot, F. Simondon, Sourse-type solutions to porous medium equations with convection, Preprint №96/17, Universite de Franche Comte.

[19] Ph. Laurencot, F. Simondon, "Sourse-type solutions to porous medium equations with convection II", Preprint N 96/33.

[20] J. I. Diaz, R. Kersner, "Non existence d'une des frontières libres dans une équation dégénerée en théorie de la filtration", C. R. Acad. Sci. Paris Ser. I Math., 296 (1983), 505-508.

[21] B.H. Gilding, "The occurrence of interfaces in nonlinear diffusion-advection processes", Arch. Ration. Mech. Anal., 100:3 (1988), 243-263.

[22] B. H. Gilding, R. Kersner, "Instantaneous shrinking in nonlinear diffusionconvection", Proc. Amer. Math. Soc., 109:2 (1990), 385-394.

[23] A.E. Shishkov, "Instantaneous shrinking phenomenon for solutions of higherdimensional nonlinear diffusion-convection equations", Methods Funct. Anal. Topology, 5:3 (1999), 54-76.

[24] M. Guedda, D. Hilhorst, C. Picard, "The one-dimensional porous medium equation with convection: Continuous differentiability of interfaces after the waiting time", Appl. Math. Lett., 5:1 (1992), 59-62.

[25] S. Bonafede, G. R. Cirmi, A. F. Tedeev, "Finite speed of propagation for the porous media equation with lower order terms", Discrete Contin. Dynam. Systems, 6:2 (2000), 305-314.

[26] Б. Гилдинг, А. Е. Шишков, "Распространение носителей решений уравнений типа многомерной нелинейной диффузии с конвекцией", УМН, 53:4 (1998), 142.

[27] Д. А. Сапронов, А.Е. Шишков, "Распространение возмущений в квазилинейных многомерных параболических уравнениях с конвективным членом", Укр. матем. журн., 53:7 (2001), 953-969.

[28] E. Zuazua, "Weakly nonlinear large time behavior in scalar convection-diffusion equations", Differential Integral Equations, 6:6 (1993), 1481-1491.

[29] S. Claudi, F. R. Guarguaglini, "Large time behaviour for the heat equation with absorption and convection", Adv. Appl. Math., 16:4 (1995), 377-401.

[30] F. Bernis, "Qualitative properties for some nonlinear higher order degenerate parabolic equations", Houston J. Math., 14:3 (1988), 319-352.

[31] О.А. Олейник, Г. А. Иосифьян, "Аналог принципа Сен-Венана и единственность решений краевых задач в неограниченных областях для параболических уравнений”, УМН, 31:6 (1976), 142-166.

\section{Д. А. Сапронов (D. А. Sapronov)}

Донецкий национальный университет,

Украина

E-mail: dsapronov@yahoo.com
Поступила в редакцию

04.01 .2003 и 13.05 .2005

\section{А. Е. Шишков (А. E. Shishkov)}

Институт прикладной математики и механики

НАН Украины, г. Донецк

E-mail: shishkov@iamm.ac.donetsk.ua 